\title{
Extensive air shower simulations at the highest energies
}

\author{
J. Knapp ${ }^{\text {a,* }}$, D. Heck ${ }^{\text {b }}$, S.J. Sciutto ${ }^{\text {c }}$, M.T. Dova ${ }^{c}$, M. Risse ${ }^{b}$ \\ ${ }^{\text {a }}$ Department of Physics and Astronomy, University of Leeds, Leeds LS2 9JT, UK \\ ${ }^{\mathrm{b}}$ Institut für Kernphysik, Forschungszentrum Karlsruhe, D-76021 Karlsruhe, Germany

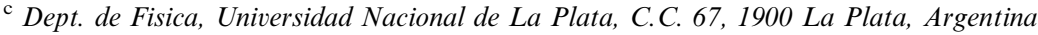

Received 30 April 2002; accepted 28 June 2002

\begin{abstract}
Air shower simulation programs are essential tools for the analysis of data from cosmic ray experiments and for planning the layout of new detectors. They are used to estimate the energy and mass of the primary particle. Unfortunately the model uncertainties translate directly into systematic errors in the energy and mass determination. Aiming at energies $>10^{19} \mathrm{eV}$, the models have to be extrapolated far beyond the energies available at accelerators. On the other hand, hybrid measurement of ground particle densities and calorimetric shower energy, as will be provided by the Pierre Auger Observatory, will strongly constrain shower models. While the main uncertainty of contemporary models comes from our poor knowledge of the (soft) hadronic interactions at high energies, also electromagnetic interactions, lowenergy hadronic interactions and the particle transport influence details of the shower development. We review here the physics processes and some of the computational techniques of air shower models presently used for highest energies, and discuss the properties and limitations of the models.
\end{abstract}

(C) 2002 Elsevier Science B.V. All rights reserved.

\section{Introduction}

The cosmic ray (CR) energy spectrum extends up to $3 \times 10^{20} \mathrm{eV}$. The presence of the highest-energy particles (ultra high-energy CRs, UHECRs) poses an enigma, since many good arguments suggest that they should not be observed. This apparent contradiction has stimulated a variety of more exotic explanations of their existence. The enigma can only be solved by an experiment that can provide a much larger event statistics than the about 20 events with $E>10^{20} \mathrm{eV}$ measured in the

\footnotetext{
${ }^{*}$ Corresponding author.

E-mail address: j.knapp@leeds.ac.uk (J. Knapp).
}

past 35 years. Knowing the form of the energy spectrum of the CR particles, their arrival direction distribution over the whole sky, and possibly even their mass composition, would allow us to test some of the hypotheses about their origin and help to identify their sources. At present experimental results suggest that UHECRs are protons or nuclei, as for CRs at much lower energies. However, many of the more exotic models of UHECR origin predict also photons and neutrinos.

The measurement of extensive air showers (EAS) is presently the only way to study CRs with energies above about $10^{15} \mathrm{eV}$. The properties of primary CRs have to be deduced from the development of the shower in the atmosphere and from the particle ratios in the shower. The incident 
direction can easily be reconstructed from the arrival times of shower particles at different positions at the observation level and the primary energy is approximately reflected in the total number of secondary particles produced. The mass of the primary particle is more difficult to measure. It is reflected, in a subtle way, in the shower form, specifically the height of the shower maximum, and in the muon-to-electron ratio of the shower.

The Pierre Auger Observatory is conceived to measure CRs with energies $>10^{19} \mathrm{eV}$ with good statistics over the whole sky [13]. It will consist of two detector sites, one in the southern and one in the northern hemisphere. Each site covers an area of $3000 \mathrm{~km}^{2}$ and combines two techniques to measure (i) the particle distribution at observation level with an array of water Cherenkov detectors and (ii) the longitudinal shower development via optical imaging of the fluorescence light in the atmosphere during clear moonless nights $(\approx 10 \%$ of the total time). This hybrid detection provides a way to inter-calibrate both sub-systems and to control systematic uncertainties. The energy determination via the fluorescence light is basically calorimetric and therefore much less modeldependent than the energy reconstruction from particle densities at ground level. The southern site is presently under construction in Argentina. The size of the site was chosen to register about 5000 events with energies $>10^{19} \mathrm{eV}$ and about $40-80$ events above $10^{20} \mathrm{eV}$ per year.

Since experiments at energies $>10^{15} \mathrm{eV}$ cannot be calibrated with a test beam the interpretation of EAS measurements is performed by comparing experimental data with model predictions of the shower development in the atmosphere. Therefore quantitative results rely on the model assumptions and on the quality of the simulation of particle interactions and transport in the atmosphere.

The detailed shower development is far too complex to be fully described by a simple analytical model. Therefore it is usually modeled by Monte Carlo (MC) simulation of transport and interaction of each individual shower particle, employing our present knowledge on interactions, decays and particle transport in matter. While the electromagnetic interaction (responsible for electromagnetic sub-showers, ionization, Cherenkov light production, ...) and the weak interaction (responsible for decays of unstable secondaries) are well understood, the major uncertainties in EAS simulation arise from the hadronic interaction models. With the present theoretical understanding of soft hadronic interactions, i.e. those with a small momentum transfer, one cannot calculate interaction cross-sections or particle production from first principles. Therefore, hadronic interaction models are usually a mixture of fundamental theoretical ideas and empirical parametrisations tuned to describe the experimental data at lower energies. The large extrapolation (over six orders of magnitude in energy) needed from experimental accelerator data to CR interactions is the second major source of uncertainty, and with an uncertain interaction model it is difficult to determine the energy spectrum and the composition of CRs.

As a consequence of the better understanding of hadronic and nuclear interactions at high energies, and the increase in computing power, shower models have improved dramatically over recent years. Also the understanding of the measuring process with a detector has markedly advanced. It is now possible to describe consistently experimental results over a wide range of energies and even to test hadronic models on the $20 \%$ level.

In this paper we review the physics and techniques of state-of-the-art modeling of EAS, specifically those important for energies of $10^{19} \mathrm{eV}$ or above. In Sections 2 and 3 we describe the modeling of hadronic and electromagnetic interactions, respectively. Section 4 summarizes briefly the simulation of showers induced by photons and neutrinos or other more exotic primary particles. Statistical thinning techniques that allow simulation of showers at the highest energies in a finite time are discussed in Section 5. Then two shower simulation programs are described and compared in Section 6. Some simulation results are shown in Section 7.

\section{Hadronic interaction models}

The highest-energy reached in a man-made accelerator is at present about $E_{\text {lab }}=900 \mathrm{GeV}$. This is 
about 8 orders of magnitude smaller than the highest-energy ever measured for a CR particle. Events triggered and examined at accelerator experiments are those that produce particles with high momentum transfer. They are well described by QCD but they constitute only a minute fraction $\left(\ll 10^{-6}\right)$ of the overall reaction rate. Interactions with low momentum transfers, i.e. soft collisions, produce particles with small transverse momenta that mostly escape undetected in the beam pipe. Of special importance are the diffractive dissociation events, which originate from rather peripheral collisions with a small fraction of energy transferred into secondary particles. But these reactions are important for air showers, since they carry the energy deep down into the atmosphere and thus drive the air shower development. Moreover, CRs collisions are predominantly nucleon-nucleus or nucleus-nucleus collisions, for which accelerator results are available only at much lower energies, rather than proton-proton collisions. Models based on accelerator results have to be extrapolated far into unknown territory in energy, in the kinematic range of very forward particle production, and to other projectile-target combinations. That is why it is of utmost importance that models rely on a sound theoretical basis which gives some guidelines on how the interactions evolve with energy.

Early hadronic models have been purely phenomenological. Accelerator results were parametrised and crudely extended to deal with diffractive interactions and nucleon-nucleus collisions. Primary nuclei have been treated with the assumption that a nucleus is just a superposition of free nucleons. Then the parametrisations have been extrapolated to high energies. It was recognised that the development of an EAS for a given energy and primary is mainly dependent on two factors: on the inelastic cross-sections $\sigma_{\text {inel }}$ of primary and secondary particles with air and on the average fraction of the available energy transferred into secondary particles (usually termed inelasticity $\left.k_{\text {inel }}\right)$. Some of the models even had $\sigma_{\text {inel }}$ and $k_{\text {inel }}$ as their main free parameters. With these simple models occasionally even very basic experimental data just could not be explained at all and therefore a proper interpretation of the data was impossible.
Over the last 10 years this situation has changed. Microscopic models have been developed that are based more and more on reliable theoretical foundations and attempt to describe not only CR showers but equally well heavy ion collision or other particle interactions. These models have in general a much smaller number of free parameters reducing the arbitrariness, and allow, to some extent, to predict $\sigma_{\text {inel }}$ as a function of energy and to deduce the average value of $k_{\text {inel }}$ as well as its distribution from deeper-lying principles.

The new models simulate interactions of nucleons and nuclei on the basis of the GribovRegge theory (GRT) [42], which most successfully describes elastic scattering and, via the optical theorem, the total hadronic cross-section as a function of energy. Table 38.2 in the particle data book [27] demonstrates good agreement for all possible scattering processes examined with the Reggeon-Pomeron scattering scheme. In GRT the observed rise of the cross-sections at high energies is a consequence of the exchange of multiple supercritical Pomerons. Inelastic processes are described by cut Pomerons leading to two colour strings each, which fragment subsequently to colour neutral hadrons. The probability of $n$ exchanged and $m$ cut Pomerons is uniquely predicted by the theory. While this is common for all implementations of the GRT, it is still debated how to realize best diffractive events and the production and decay of colour strings. Presently the GribovRegge approach is the only theoretically sound way, and also the most successful one, to model energetic soft hadronic interactions. A good introduction to GRT models is given in Ref. [70]. At present GRT-type models used for CRs are QGSJET [50,51], VENUS [70], DPMJET [63], and, most recently, NEXUs [33].

Present work on GRT models is focused on the consistent treatment of diffractive reactions, by inclusion of specific, higher order Pomeron exchange diagrams that account for single and double diffraction, and the inclusion of hard processes, as described by QCD, that become more important with energy. Experiments at HERA have improved our knowledge of the parton momentum distribution function inside a nucleon (see e.g. Ref. [4]) which allow for a quantitative 
account of the effect of hard interactions. This is vital when extrapolating cross-sections and particle production up to the highest energies. At present only QGSJET, DPMJET and SIBYLL reach up to $>10^{20} \mathrm{eV}$.

NEXus, the newest of the models, is constructed as a combined effort of the authors of VENUS and QGSJET to model hadronic interaction to the best of our knowledge, and to combine all necessary parts in a consistent way. They start from the Universality hypothesis stating that the mechanisms of high-energy interactions are identical in different type reactions. Thus one can study the final state parton evolution and the subsequent hadronisation on the basis of $\mathrm{e}^{+} \mathrm{e}^{-}$ data, while the initial parton cascade can be tuned to deep-inelastic lepton-proton data. In this way the hadronic interaction is broken into separate building blocks which are deduced from simpler systems. This constrains considerably the model parameters and ensures a more reliable extrapolation. NEXUs is by construction a GRT-type model with unified soft and hard interaction. The former is described by the traditional soft Pomeron exchange, the latter uses perturbative QCD within the concept of the semi-hard Pomeron. In addition NEXUs employs a fully self-consistent treatment of energy and momentum sharing between individual elementary scattering processes.

A model somewhere in between the purely phenomenological ones and the GRT-type models is SIBYLL [37]. SIBYLL 1.6 was a so-called minijet model, inspired by QCD, and treated the soft part rather crudely. It simulated a hadronic reaction as a combination of an underlying soft collision, in which two strings are generated, and a number of minijet pairs leading to additional colour strings with higher transverse momentum ends. In SIBYLL 1.6 the rise of the cross-section with energy was solely due to the minijet production. Recently the soft part of SIBYLL was revised to allow for multiple strings from the soft collision, leading to a contribution of soft interactions to the rise of the cross-section, and for an energy dependent transverse momentum cut-off for the minijet production [35]. With the new SIBYLL 2 a much better agreement of model predictions with experimental results is achieved.
Cross-sections for nucleus-nucleus interactions are calculated on the basis of the geometrical Glauber model [41]. However, to simulate particle production properly requires, for each projectile nucleon, knowledge how often and with which of the target nucleons it interacts. In more recent models this is determined via explicit tracking of the nucleons in projectile and target during the collision.

The decay of colour strings into observable hadrons, as in particle physics, is still a phenomenological procedure, but there is no reason to assume that colour strings in CR showers should decay differently from those from $\mathrm{e}^{+} \mathrm{e}^{-}$or $\mathrm{p} \overline{\mathrm{p}}$ reactions. Therefore those algorithms can be adopted that have been proven by particle physics experiments.

Also the low-energy hadronic interactions are difficult to model. While for energies around 100 $\mathrm{GeV}$ many details are known about hadronic and nuclear interactions, in the $\mathrm{GeV}$ range, where low particle multiplicities dominate and resonances are important, only phenomenological models exist. Since most of the particles observed in a CR experiment stem from interactions in the low-energy regime, these models can influence predictions, too. One program for low-energy hadronic interactions is GHEISHA [36], which was written around 1985 to simulate the interactions of $\mathrm{GeV}$ secondaries from $\mathrm{e}^{+} \mathrm{e}^{-}$collisions with typical detector materials. GHEISHA is a phenomenological model, i.e. it was tuned to experimental results for a variety of projectiles and targets in the few$\mathrm{GeV}$ region and, consequently, reproduces crosssections and particle production rather well. It is also used within the CERN detector simulation package GEANT 3 [24].

As an alternative program to GHEISHA for the low-energy hadronic interactions the ultrarelativistic quantum molecular dynamics program UrQMD [18] is considered which is developed and used for heavy ion collision experiments. With it more detailed and theory-based calculations can be performed, than with GHEISHA, in the few$\mathrm{GeV}$ energy region.

A simpler, but very fast, approach is pursued by the Hillas splitting algorithm (HSA) [46] in which the initial energy is split at random into smaller 
and smaller portions. The secondary particles are created from these energy packets, assigning their identity according to externally provided probabilities. In spite of its simplicity, the HSA can be tuned to emulate very well some of the characteristics of the secondaries emerging from hadronic collision, e.g. their multiplicity and energy distributions. However, the HSA has to be complemented with additional procedures to assign identity and transverse momentum to the secondaries, and also the corresponding cross-sections must be provided externally. The HSA can be adequately configured to reproduce the main results from other, generally more involved, models.

Since GHEISHA was tuned in the few-GeV region and QGSJET uses the Gribov-Regge approach, which works fully only in the range $E_{\text {lab }}>$ $100 \mathrm{GeV}$, the two models do not fit together smoothly. The difference between the models $(\approx 25 \%)$ is usually well within the fluctuations for a single interaction and the flexibility of HSA could be used to interpolate between models for low and
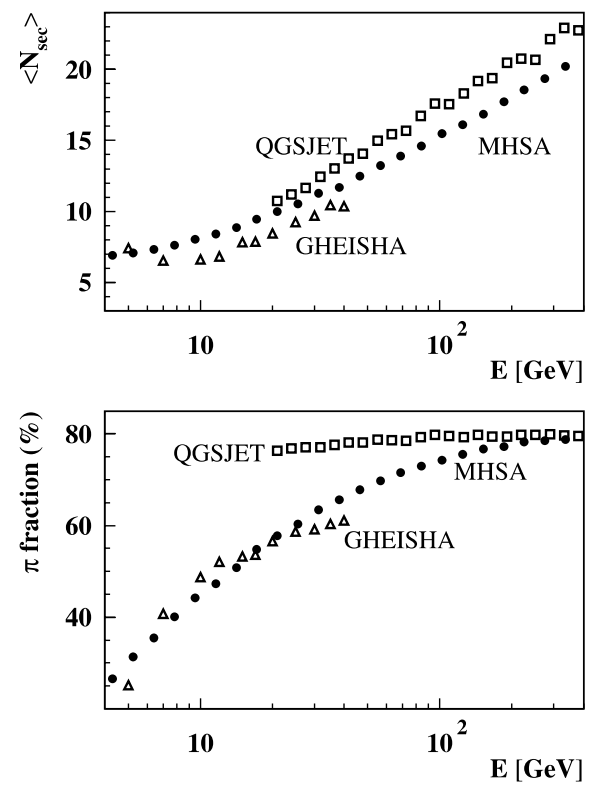

Fig. 1. Average number of secondaries and fraction of pions (charged + neutral) versus primary laboratory energy in proton-air collisions. The triangles and squares correspond to GHEISHA and QGSJET, respectively, and the full circles show a (modified) HSA tuned to reproduce GHEISHA in the few$\mathrm{GeV}$ range and QGSJET far above $100 \mathrm{GeV}$. high energies in the intermediate range where both are stretched to their limits. This is illustrated in Fig. 1. However, this example demonstrates also that the HSA has no predictive power on its own and needs a more advanced model according to which its parameters are adjusted.

It holds in general, that the more complex a model is, the longer the computing times become. Especially in an air shower cascade, where many millions of hadronic interactions occur, this may be prohibitive. Therefore, two different approaches may coexist: reference-like models that do the simulations as well as possible without consideration of computing time, and faster but simpler models that are tuned in a first step to reproduce the results of the reference.

\subsection{Inelastic cross-sections}

A summary of experimental and predicted cross-sections is shown in Fig. 2. In 1997 the variation of the p-air cross-section in experiments as well as in models amounted to about $25 \%$ at $3 \times 10^{15} \mathrm{eV}$, and to about $40 \%$ at $10^{18} \mathrm{eV}$ (shown in the upper panel). For nucleus-air cross-sections the model uncertainty was only $10-15 \%$ due to the averaging effect of many nucleons inside the nucleus. After some of the models have been revised new theoretical predictions of cross-sections have been published. The situation is shown in the lower panel of Fig. 2. Some of the models shown in the upper plot are no longer pursued by their respective authors.

Recent calculations of the $\mathrm{p}$-air cross-sections from Frichter et al. [39] agree well with the QGSJET cross-sections up to about $10^{17} \mathrm{eV}$. Beyond this energy the predictions become significantly higher than those of QGSJET, similar to the values of SIBYLL. Also Block et al. [23] performed new calculations by using a QCD inspired parameterization of accelerator data. They fitted simultaneously the total $\mathrm{p}-\mathrm{p}$ cross-sections, the ratio of real to imaginary part of the forward scattering amplitude $\rho$, and the nuclear slope parameter B. Then $\mathrm{p}-\mathrm{p}$ cross-sections were converted via Glauber theory into $\mathrm{p}$-air cross-sections. Their extrapolation is shown in Fig. 2(b) and agrees very well with the cross-sections used in the QGSJET model. 


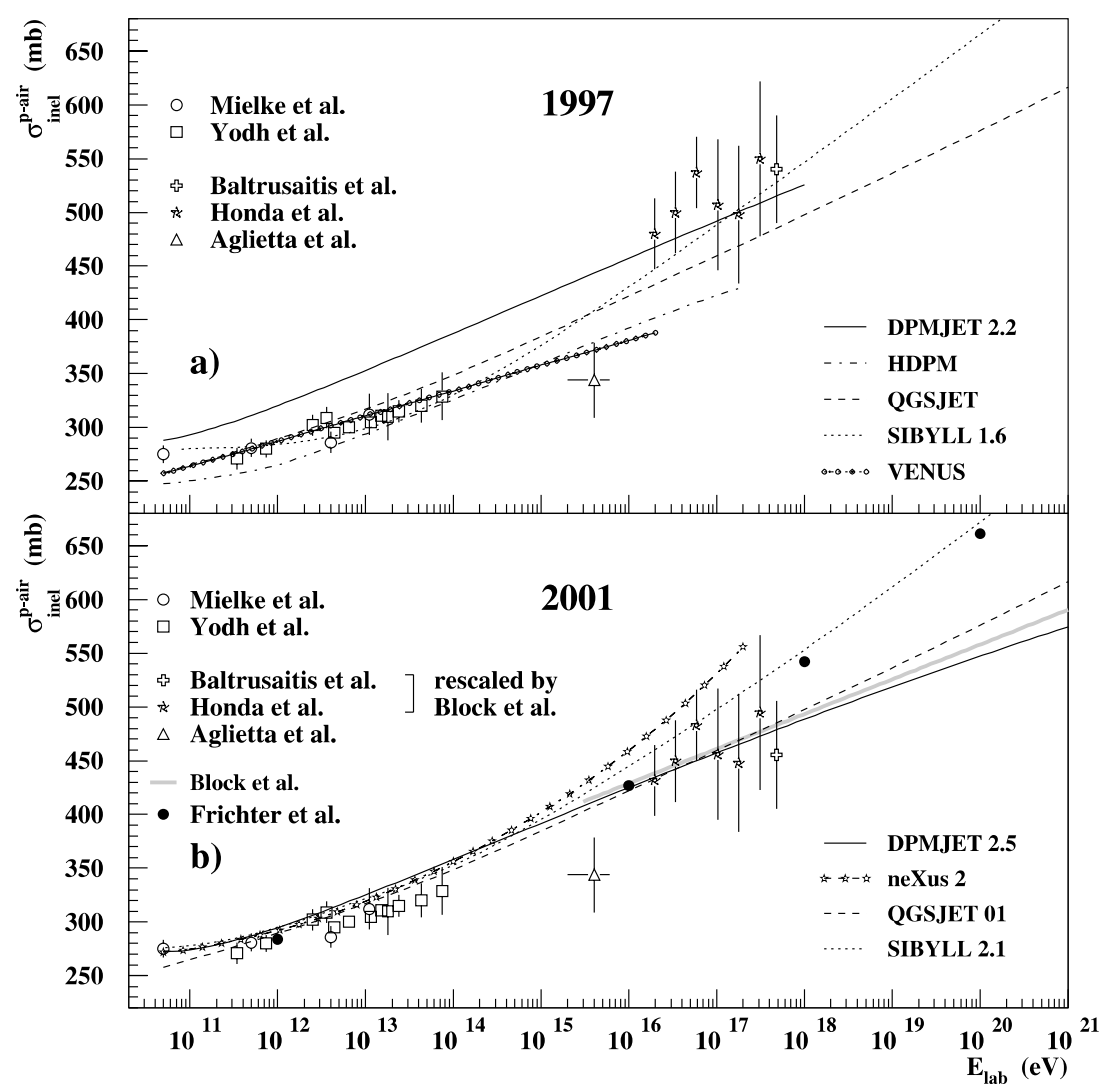

Fig. 2. Inelastic p-air cross-sections from measurements and models. Upper panel: situation in 1997. Lower panel: situation in 2001. Experimental data are taken from Refs. [5,17,49,59,71], theoretical calculations from Refs. [23,39].

To extract cross-sections directly from EAS measurements is not possible. Usually the attenuation length $\Lambda$ of EAS in the atmosphere is measured. Experiments select deeply penetrating showers to retain only proton showers in their sample. The attenuation length relates to the crosssections $\sigma_{\text {inel }}^{\mathrm{p} \text {-air }}$ via $\Lambda=14.6 \cdot k \cdot m_{\mathrm{p}} / \sigma_{\text {inel }}^{\mathrm{p} \text {-air }}$, where $m_{\mathrm{p}}$ is the proton mass and $k$ depends strongly on the average inelasticity and the inelasticity distribution of $p$ and $\pi$ reactions and on the energy. While early and naive models fixed the inelasticity as one of the free parameters, in GRT models the inelasticity distribution emerges inevitably as a result of more fundamental properties of the interaction model. Since a value of $k$ has to be assumed to derive the experimental cross-sections, the final results are biased. In addition, different groups have been using different values for $k$. The experimental re- sults can be easily brought into agreement with each other and with the theoretical calculations by slightly modifying $k$ [23]. For AGASA and Fly's Eye data such a correction brought the experimental values in the energy range $10^{16}$ to $10^{18} \mathrm{eV}$ down by $12-20 \%$, in good agreement with the cross-sections as predicted by Block et al. or by QGSJET.

As a result of various modifications the model predictions at knee energies (i.e. $\approx 3 \times 10^{15} \mathrm{eV}$ ) now agree to within about $6 \%$.

\subsection{Particle production}

The second important quantity that rules the shower development is the inelasticity. This quantity combines the multiplicity and the energy of the secondaries, thus describing how much of the 
energy of the incoming particle is transferred onto secondary particles. Therefore it is more relevant than the particle multiplicity alone. High inelasticity means that the energy is dissipated quickly and the shower develops fast, i.e. it reaches its maximum higher up in the atmosphere. Low inelasticity means that the leading particle carries off most of the energy, leading to slow developing and long showers. Obviously inelasticity and crosssection influence the shower development in a very similar way, and are therefore difficult to disentangle. In Fig. 3 the distributions of the longitudinal momentum fraction, $x_{\mathrm{lab}}$, that is carried away by the most energetic baryon in $\mathrm{p}-{ }^{14} \mathrm{~N}$ collisions are shown. $x_{\mathrm{lab}}$ relates directly to the inelasticity via $k_{\text {inel }}=1-x_{\text {lab }}$. It is obvious from the figure that the average value of $k_{\text {inel }}$ alone does not fully describe the distribution. Indeed, the fluctuation in $k_{\text {inel }}$ is one of the major sources of shower fluctuations. The distinctive peak near $x_{\text {lab }}=1$ represents the diffractive events and reactions with large $x_{\text {lab }}$ are the ones that carry the energy efficiently deep into the atmosphere. Since accelerator experiments barely see diffractive events and their theoretical treatment allows some freedom, the models exhibit a large spread in the range $x_{\text {lab }}>0.8$, which directly translates into a systematic uncertainty in the shower analysis (see, for instance, Ref. [11]). It is hoped that new experiments at LHC or RHIC will measure particle production in the very forward direction and thus help to reduce this uncertainty. The overall form of the elasticity distributions is almost independent of the collision energy and their average values change only slowly with energy. All models show a decrease (see Fig. 4) from about 0.5 at $E_{\text {lab }}=10^{12}$ $\mathrm{eV}$ to about 0.25 at $10^{20} \mathrm{eV}$. The GRT-type models predict lower values than SIBYLL.

In contrast to a widely-held belief the multiplicity of an hadronic interaction on its own is not of great importance for the shower development. Only the high-energy secondaries can influence the shower development markedly, but secondaries of very low energies are irrelevant. This can be demonstrated impressively by comparison of QGSJET with DPMJET and SIBYLL. The average charged multiplicities of $\pi-\mathrm{N}$ collisions at $E>10^{19} \mathrm{eV}$ differ by about a factor 5 (see Fig. 5) due to QGSJET interactions having a tail to very high numbers of secondaries which grows with energy. This is shown in Fig. 6 for $\pi-\mathrm{N}$ collisions at $E_{\pi}=10^{15}$ and $10^{19} \mathrm{eV}$. Nevertheless the fraction of energy converted into secondaries for QGSJET is in between the values for SIBYLL and DPMJET (see Fig. 4). The reason is that most of the secondaries produced in QGSJET have low

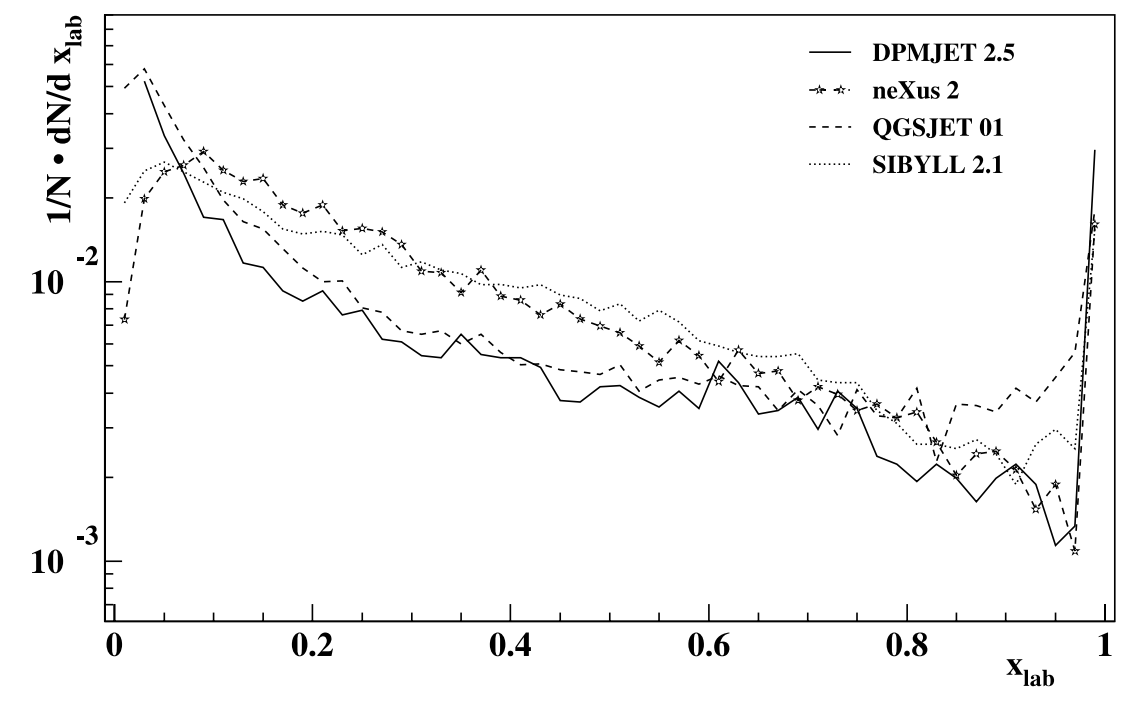

Fig. 3. Distributions of longitudinal momentum fraction carried away by the leading baryon emerging from $\mathrm{p}-{ }^{14} \mathrm{~N}$ collisions at $E_{\mathrm{lab}}=10^{7} \mathrm{GeV}$. 


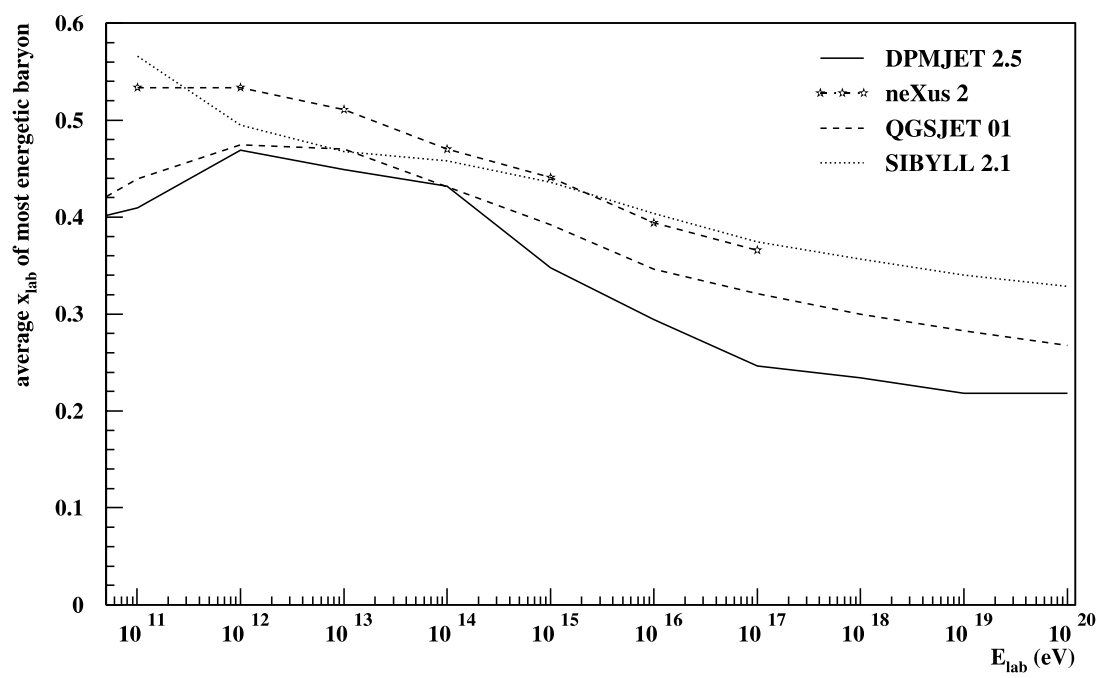

Fig. 4. Average of longitudinal momentum fraction carried away by the leading baryon emerging from $\mathrm{p}-{ }^{14} \mathrm{~N}$ collisions as function of energy.

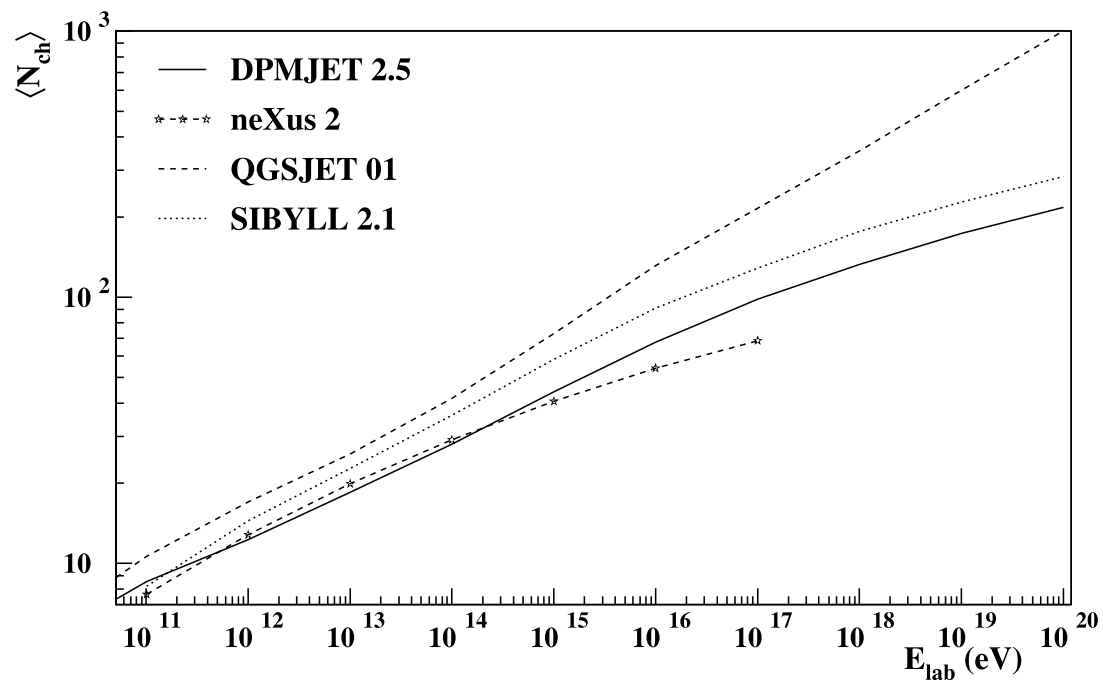

Fig. 5. Average charged multiplicity in $\pi-{ }^{14} \mathrm{~N}$ collisions as a function of energy as predicted by various models.

energies and are produced in the central region rather than in the forward region which is most important for the shower development. Also the resulting longitudinal distributions of shower particles, as discussed in the following section, show only relative small differences between QGSJET, DPMJET and SIBYLL at $10^{19} \mathrm{eV}$ (Fig. 8).

\subsection{Air shower development}

The cross-section and the inelasticity determine the longitudinal development of an EAS, which in turn is closely related to the most important shower observables: the particle number at ground level and their lateral distribution, the height of 

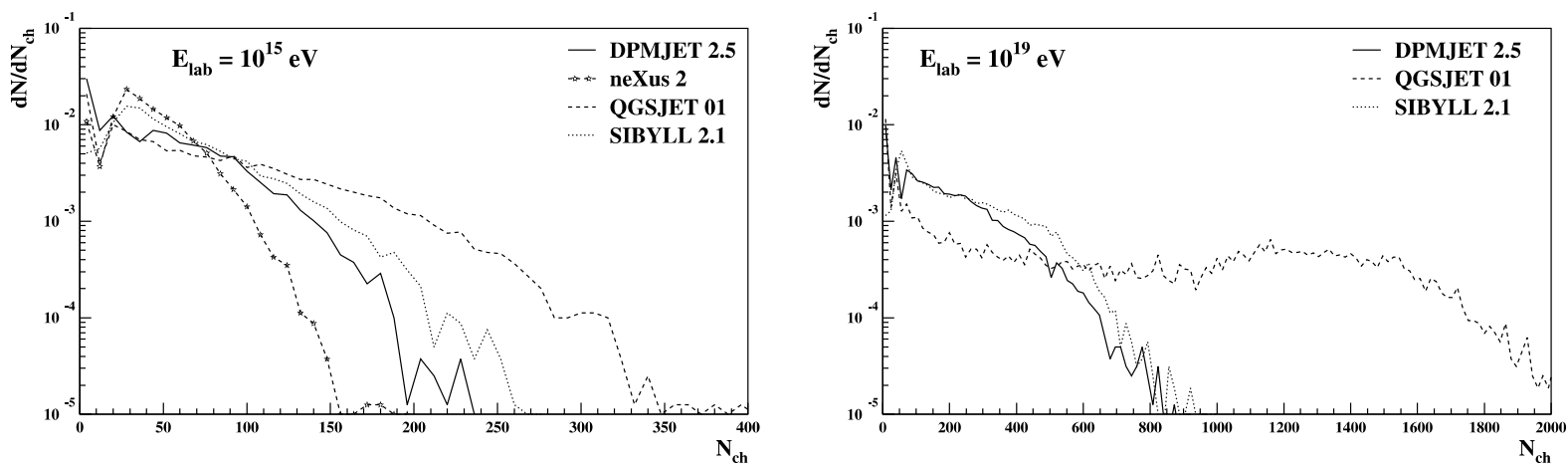

Fig. 6. Distribution of charged multiplicity of $\pi-^{14} \mathrm{~N}$ collisions at $10^{15}$ and $10^{19} \mathrm{eV}$ as predicted by various models.

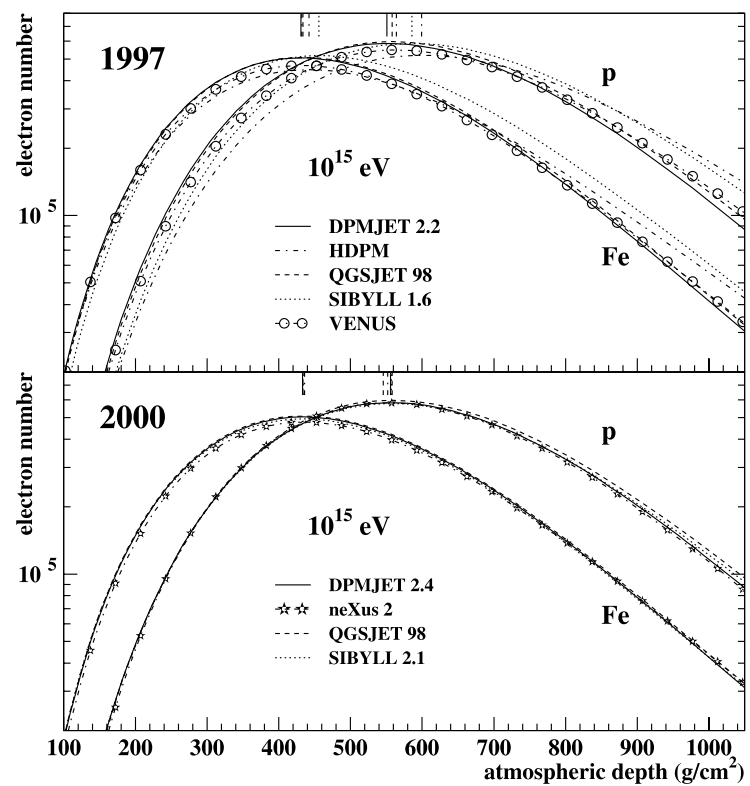

Fig. 7. Average longitudinal shower development of vertical $10^{15} \mathrm{eV}$ showers. The positions of the shower maxima are indicated by ticks at the upper rim of the picture. Upper panel: situation in 1997. Lower panel: situation in 2000.

shower maximum $X_{\max }$, and the total energy deposited in the electromagnetic component. In Fig. 7 average shower curves of $10^{15} \mathrm{eV}$ showers from different models are shown. The model convergence between 1997 and 2000 is clearly visible. While for the 1997 models the position of $X_{\max }$ varied by about $7.5 \%$ for protons and $5.5 \%$ for iron showers, with the new models the variations are below $1 \%$. Also the particle numbers at ground

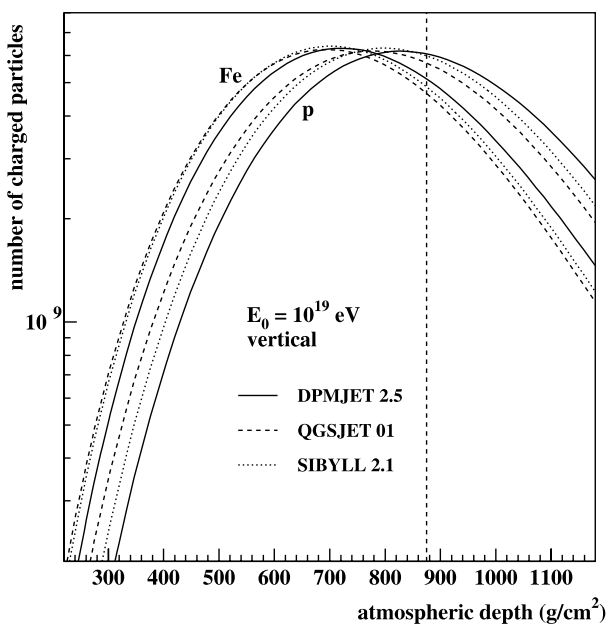

Fig. 8. Average longitudinal shower development for vertical proton and iron showers of $10^{19} \mathrm{eV}$. The dashed vertical line indicates the atmospheric depth of the Auger site.

level agree much better than a few years ago. The variations for $\mathrm{p}$ and $\mathrm{Fe}$ showers were reduced from $80 \%$ and $50 \%$ to $15 \%$ and $5 \%$, respectively. Although $10^{15} \mathrm{eV}$ is far below the energies relevant for the Auger Observatory, Fig. 7 illustrates the spread where more hadronic models are available. A detailed comparison of SIBYLL 1.6 and QGSJET 98 at energies $>10^{19} \mathrm{eV}$ has been reported in Ref. [9], where it is shown that the systematic uncertainty is markedly larger. As illustration the shower curves of $10^{19} \mathrm{eV}$ proton and iron showers as obtained with the latest version of three models are presented in Fig. 8. The difference between the models is much larger, though it is still smaller 

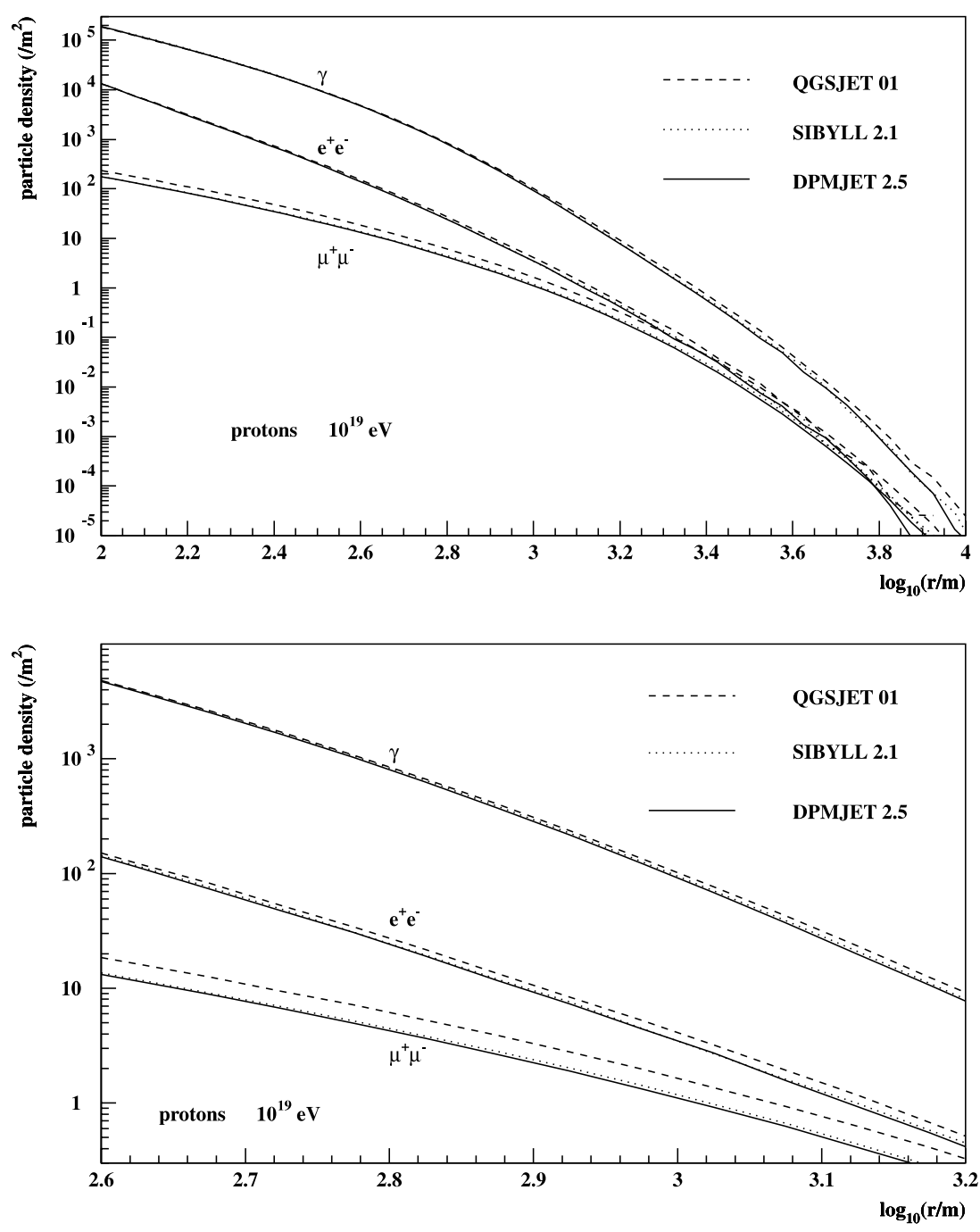

Fig. 9. Average lateral distributions of photons, electrons and muons in vertical proton showers of $10^{19} \mathrm{eV}$. The lower panel shows the distance range from 400 to $1600 \mathrm{~m}$ enlarged.

than the expected difference between proton and iron showers. The SIBYLL 2.1 curve falls right between the predictions for two GRT-type models.

Fig. 9 shows the lateral distributions of secondary photons, electrons and muons in protoninduced showers of $10^{19} \mathrm{eV}$ for different models, in the radial distance range where Auger will record data.

DPMJET 2.5 and SIBYLL 2.1 distributions agree to better than $5 \%$ for all secondaries. QGSJET predicts systematically higher particle densi- ties, due to the long tail in the multiplicity distributions (see Fig. 6). Also the lateral distributions are slightly flatter (Fig. 9). At a core distance of $1 \mathrm{~km}$ the photon and electron densities differ by about $10-15 \%$ and the muon densities by about $30 \%$. For Auger this corresponds directly to an uncertainty in the energy determination from the surface detectors of about $20 \%$. The crosscalibration of surface detectors and fluorescence detector will perhaps allow to discriminate models at this level of precision. 
Also the models used for low-energy hadronic interactions introduce a systematic uncertainty, e.g. with UrQMD there are about $30 \%$ more baryons in the energy range $2-10 \mathrm{GeV}$ and factor of 2 less for $0.1-2 \mathrm{GeV}$ than with GHEISHA. Also UrQMD tends to produce $10 \%$ more muons below $4 \mathrm{GeV}$ and $10 \%$ less above $4 \mathrm{GeV}$, the total number of muons stays approximately the same in both cases. As mentioned above, the predictions of the HSA depend sensitively on the parameter setting, e.g. the number of muons with $E_{\mu}<10 \mathrm{GeV}$ can vary by up to $40 \%$.

The direct observation of the shower curve and the shower maximum is possible with detectors that register the fluorescence or Cherenkov light produced by the shower particles. These measurements are of great advantage for the reconstruction of the shower energy and the primary mass, but have a reduced duty cycle since they require clear and moonless nights. The Fly's Eye experiment pioneered this technique and measured $X_{\max }$ as a function of energy (see Fig. 10). When first published there were difficulties in reproducing the experimental data with the available model predictions. The Fly's Eye Collaboration interpreted their data by comparing to simulations from Gaisser et al. [40]. The MC predictions, however,

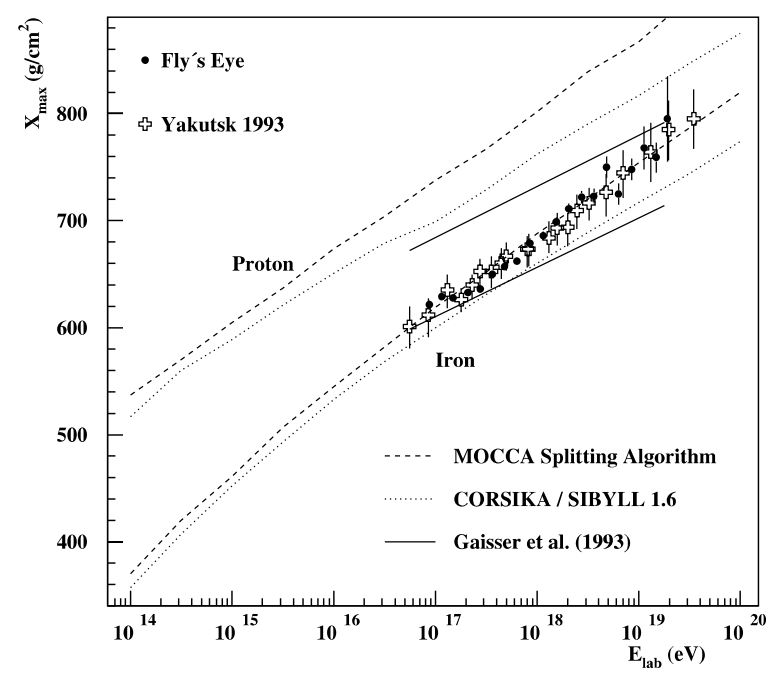

had to be shifted arbitrarily by $25 \mathrm{~g} / \mathrm{cm}^{2}$ to avoid particles heavier than iron. Also the change of $X_{\max }$ with energy, i.e. the elongation rate, was different in data and simulation. The different elongation rate led to the conclusion that the $\mathrm{CR}$ composition changed from basically pure $\mathrm{Fe}$ at $2 \times 10^{17} \mathrm{eV}$ to pure protons at $2 \times 10^{19} \mathrm{eV}$ (see left panel of Fig. 10). When compared to MOCCA simulations using the HSA up to highest energies the Fly's Eye data would have suggested a CR composition of iron or even heavier. However, it later became clear that the elongation rate, and therefore the interpretation of the data, was strongly model dependent $[51,30]$.

Meanwhile a number of experiments have provided data on $X_{\max }$ as a function of energy and the results are summarised in the right panel of Fig. 10. More recent models can reproduce the absolute values of $X_{\max }$ over the whole energy range and the change of mass composition seems much more moderate over the Fly's Eye energy range. However, a spread of the models of about $20-50 \mathrm{~g} / \mathrm{cm}^{2}$ for iron and proton showers, respectively, persists.

Close inspection of prediction of $X_{\max }(E)$ from the modern models (e.g. QGSJET $01[50,51,45])$ reveals that the superposition assumption, employed by some of the models to simulate nuclear

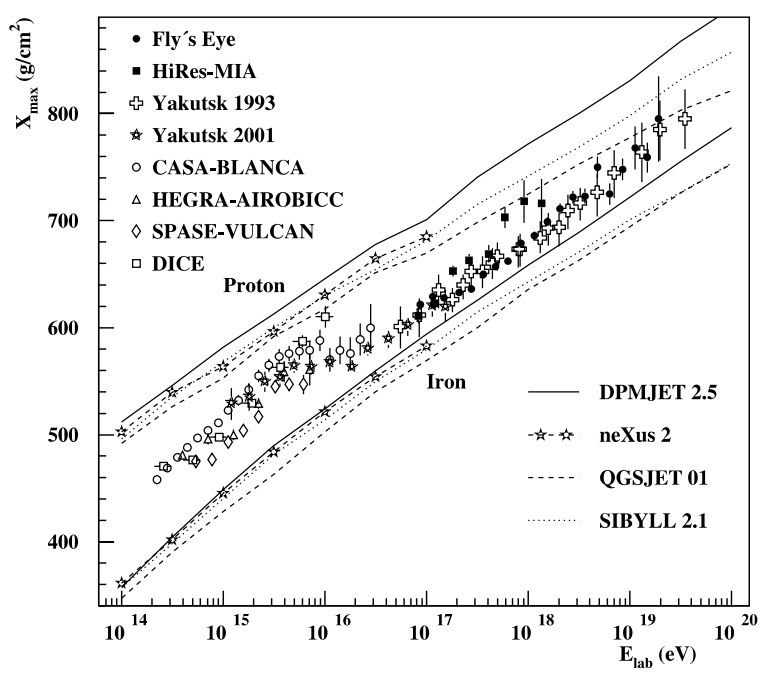

Fig. 10. Shower maximum $X_{\max }$ as function of energy. Left panel: measurements from Fly's Eye [22] and Yakutsk [34], compared with several models from the early 1990s [40,47,62]. Right panel: measurements, including newer data $[2,3,12,22,32,38,55,68]$, compared with up-to-date models. 
primaries, does not hold strictly. Especially for energies $>10^{16} \mathrm{eV}$ the elongation rate of ironinduced showers is larger than the one for proton showers $\left(\approx 62\right.$ and $56 \mathrm{~g} / \mathrm{cm}^{2}$ per decade, respectively). This is to be expected when the interactions of individual nucleons in a nuclear collisions are treated correctly. If more than one projectile nucleon interacts with the same target nucleus, all but the first nucleon will encounter a target that is excited above its ground state. The centre-of-mass energy of the collision, and consequently the secondary particle production, is smaller than in a superposition model where, by definition, each projectile nucleon interacts with an undisturbed target nucleus.

\subsection{Collider results}

Another, and sometimes underestimated, source of uncertainties are the errors of the collider results which are used to tune the interaction models. There are a few instances where the uncertainty of these results is revealed.

The inelastic $\mathrm{p}-\overline{\mathrm{p}}$ cross-sections have been measured by three different experiments at Fermilab $[1,8,16]$. Their values at $\sqrt{s}=1800 \mathrm{GeV}$ or $E_{\text {lab }}=1.7 \times 10^{15} \mathrm{eV}$ vary from $80.03 \pm 2.24$ to $71.71 \pm 2.02 \mathrm{mb}$. This corresponds to $12 \%$ uncertainty at an energy before the knee and clearly much more for the highest-energy CRs.

A second example is the pseudorapidity distribution of secondary particles produced in $\mathrm{p}-\overline{\mathrm{p}}$ collisions. The UA5 measurements from $\sqrt{s}=200$ to $900 \mathrm{GeV}$ [7] have been widely used to tune hadronic interaction models. GRT models always had severe difficulties to reproduce the pseudorapidity densities $\mathrm{d} N / \mathrm{d} \eta$ near $\eta=0$ and the slope in $\mathrm{d} N / \mathrm{d} \eta$ at around $\eta=4$ at the same time. However, more recent measurements by Harr et al. [43] show a clearly flatter distribution than the UA5 ones, that fits the GRT predictions very well [53]. In the near-forward region $(\eta \approx 4)$ the differences in the measured particle densities amount to about $25 \%$.

Until new experimental data permit to clarify discrepancies like the ones mentioned here, it seems rather unlikely that the basis of hadronic models, and therefore their predictions, can become much better than they are at present.

\section{Electromagnetic interactions}

In contrast to the hadronic particle production, the electromagnetic interactions of shower particles can be calculated very precisely from quantum electrodynamics. Therefore electromagnetic interactions are not a major source of systematic errors in shower simulation. Moreover there exist very well tested packages to simulate these reactions in great detail, such as EGS4 [61] or the electromagnetic parts of GEANT [24]. At the highest energies, however, a variety of effects become important that are negligible at lower energies and are usually not treated in the above mentioned programs.

High-energy photons can readily produce $\mu^{+} \mu^{-}$ pairs or hadrons. The latter process may make a photon-induced shower look more like hadronic ones. The muon pair production is calculated identically to $\mathrm{e}^{+} \mathrm{e}^{-}$pair production. The higher muon mass leads to a higher threshold for this process. QED firmly predicts the crosssection as function of energy which approaches the Bethe-Heitler cross-section reduced by the factor $\left(m_{\mathrm{e}} / m_{\mu}\right)^{2}$. The cross-section for hadron production by photons is much less certain, since it involves the hadronic structure of the photon. It has been measured at the HERA storage ring for photon energies corresponding to $E_{\gamma, \text { lab }}=2 \times 10^{13} \mathrm{eV}$ [6,31]. This energy is still well below Auger energies, but the experimental cross-sections constrain the parameterisation used for extrapolation from the $100 \mathrm{GeV}$ range. At these energies the crosssection for the process $\gamma \rightarrow \mathrm{e}^{+} \mathrm{e}^{-}$is $\approx 650 \mathrm{mb}$, i.e. much larger than the cross-sections for hadronic interaction $(\approx 1.4 \mathrm{mb})$ or for muon-pair production $(\approx 0.015 \mathrm{mb})$.

Another effect to be considered in high-energy shower simulations is the Landau Pomeranchuk Migdal (LPM) effect [57]. It describes an interference effect between particle emission and scattering that occurs only in matter. If the emission angle of the secondary particle is smaller than a typical scattering angle, the secondary particle can be readily re-absorbed. This leads to reduced cross-sections of pair production by photons, and bremsstrahlung by electrons, at very high energies, and to an effective prolongation of the radiation length, which governs the mean free path of elec- 
trons and photons and determines the development of electromagnetic showers. The production of pairs of secondaries of about equal energies is strongly suppressed. In dense media, like iron or lead, the LPM effect distorts electromagnetic showers from about $10 \mathrm{TeV}$ upwards. However, since the upper atmosphere is very thin the LPM effect becomes noticeable only for photons and electrons of more than $10^{18} \mathrm{eV}$. In hadronic showers the LPM becomes important at about $10^{20} \mathrm{eV}$, since secondary photons produced in the first interaction rarely have energies of more than $1 \%$ of the energy of the primary particle. Due to the LPM effect electromagnetic sub-showers become longer and fluctuate more than normal electromagnetic sub-showers. The proper simulation of the LPM effect is of special importance if one attempts to determine the elemental composition from the longitudinal shower profile, or if one assumes that the primary $\mathrm{CR}$ particles may be photons, as many of the scenarios that have been constructed to explain the origin of highest-energy CRs predict. For a recent discussion of the LPM effect in the context of highest-energy air shower simulation see Ref. [28].

Muons of high energies initially lose energy through $\mathrm{e}^{+} \mathrm{e}^{-}$pair production and bremsstrahlung, at approximately equal rates. The energy loss is proportional to the muon energy and in air these processes dominate the total muon energy loss only for $E_{\mu}>20 \mathrm{TeV}$. In $10^{20} \mathrm{eV}$ air showers the fraction of these high-energy muons is well below $1 \%$ of the total muon number. For a recent account of muon bremsstrahlung and pair production at Auger energies see Ref. [29]. High-energy muons also lose energy due to hadronic reactions, though at a lesser rate than through bremsstrahlung and pair production. The hadronic energy loss is the least wellknown of all reactions of muons. All these processes influence mostly the muonic component of the shower. They are therefore more important in cases where the muon component is dominant, i.e. for very inclined showers $\left(\theta>70^{\circ}\right)$ where very late stages of shower development are observed, and where most of the electromagnetic and hadronic shower particles have been absorbed. For nearvertical hadronic showers the above mentioned effects do not play a major role.
The spread of the shower particles at ground level is dominated by multiple Coulomb scattering of charged particles off the nuclei in the atmosphere. Air shower detectors for $10^{20} \mathrm{eV}$ showers must be of huge size and arrays of particle detectors are, for financial reasons, built as sparse as the spread of shower particles allows. Consequently, the array detectors usually measure only particle densities far away from the core (only 15\% of the shower cores fall within distances $<300 \mathrm{~m}$ from an Auger array detector). The particle density at large core distances is determined by the tails of the multiple scattering distribution. Molière theory of multiple scattering predicts realistic distributions, with a roughly Gaussian distribution for small scattering angles, but larger tails from single Coulomb scattering processes (for a short summary see Section 23 in Ref. [27] and references therein).

As mentioned above, the measurement of fluorescence and Cherenkov light in air, emitted by shower particles, allows a relatively reliable energy determination of the primary particle. In the Auger experiment this is used for the energy calibration of the particle array with fluorescence measurements.

For lower-energy showers it is possible to generate and track explicitly the photons produced, but for the highest-energy cascades it is not practical to propagate individual fluorescence or Cherenkov photons since the number of normal shower particles already exceeds by far what can be handled easily. Therefore, during the shower simulation distributions of shower particle location, energy and direction, as well as energy deposits are recorded at various depths in the atmosphere [65], which are used, in a second step, to simulate the light production of an individual shower, the attenuation and scattering in the atmosphere, and the image recorded at a fluorescence detector site.

\section{Non-standard primaries and interactions}

The unknown origin of highest-energy CRs has led to a variety of more or less speculative explanations. They involve yet unknown decaying superheavy particles, known particles with 
non-standard properties, or even exotic new particles. Therefore, shower simulations need to accommodate primaries other than the classical protons and nuclei. Potential candidates are UHECR photons and neutrinos, which may be produced in $\mathrm{CR}$ accelerators or as secondaries in the decays of cosmic strings or superheavy relic particles [26], neutrinos with hadron-like crosssections, monopoles, UHERONs or other exotic particles, for which cross-sections and reaction processes have to be assumed.

If the highest-energy CRs indeed were photons then also reactions of the primary photon with the Earth's magnetic field need to be considered. Photons with $E>10^{19} \mathrm{eV}$ have a large probability to convert into an $\mathrm{e}^{+} \mathrm{e}^{-}$pair well before reaching the atmosphere [58]. The pair then strongly radiates in the field and produces a number of lowerenergy photons which again may convert into a pair. A pre-shower is formed which will look markedly different in the atmosphere than a shower from a single photon of the same energy. It will be wider, since the pre-shower photons could spread out, and it will have a shorter longitudinal development since the individual sub-showers are of lower-energy and do not suffer from LPM effect. The formation of such pre-showers, however, is usually not part of the shower simulation. They are simulated with a suitable pre-processor that delivers the particles which enter the atmosphere. These are then used as inputs of the traditional air shower simulation programs [20].

Because experiments like Auger survey such a huge volume of atmosphere and because neutrino interaction cross-sections grow with energy, there is sensitivity to showers induced by high-energy neutrinos [26,19]. The experimental signature would be normal electromagnetic or hadronic showers with the exception that they start very deep in the atmosphere. Especially for nearly horizontal showers, where the atmosphere is $\approx 36000 \mathrm{~g} / \mathrm{cm}^{2}$ thick, no other primary particle can penetrate the atmosphere to initiate a high-energy shower close to the detector and a selection of neutrino-induced showers seems possible. Also neutrino reactions are usually not part of shower simulation programs. Charged or neutral current neutrino-nucleon or neutrino-electron interac- tions must be simulated externally and the resulting particles can then be fed into the shower simulation. At present some of these generators are prepared to investigate neutrino-induced showers in the Auger detector.

Additionally more exotic particles, such as magnetic monopoles or supersymmetric particles, are discussed as primaries. In addition, the first few interactions of conventional primaries may be energetic enough to produce quark-gluon plasma or other exotic states with fundamentally different number and energies of secondaries produced. To simulate these particles or reactions specific models must be constructed and implemented in the shower simulation programs, e.g. by a pre-processor.

\section{Thinning}

For highest-energy air showers the number of secondaries becomes so large $\left(>10^{11}\right)$ that it is prohibitive in computing time and disk space to follow all of them explicitly and store the ones reaching ground level. (To simulate a $10^{20} \mathrm{eV}$ shower fully would take about 10 years per shower on a $750 \mathrm{MHz}$ Linux workstation.) Therefore, the so-called statistical thinning was introduced by Hillas [46], which is a key concept in EAS simulations. A small, but representative, fraction of secondaries are fully tracked and all others are discarded. To account for the energy of the discarded particles statistical weights are assigned to the tracked particles. Thinning is invoked whenever new particles are generated. If $E_{A}$ is the energy of a particle producing secondaries of energy $E_{B_{i}}$, and $E_{\text {th }}$ is a fixed energy, called the thinning energy, then each particle in the production vertex is selected for further tracking with a probability $P_{i}$ as follows: If the energy $E_{A}$ of the primary particle is greater than $E_{\mathrm{th}}$, then the probability $P_{i}=1$ for $E_{B_{i}} \geqslant E_{\text {th }}$ and $P_{i}=E_{B_{i}} / E_{\text {th }}$ otherwise. On the other hand if $E_{A}$ is smaller than $E_{\mathrm{th}}$, meaning that the primary particle comes from a previous thinning operation, then only one of the secondaries is selected with probability $P_{i}=E_{B_{i}} / \sum_{j=1}^{n} E_{B_{j}}$. The weight of the accepted secondary particles is given by the weight of the primary particle in the vertex divided by $P_{i}$. This method ensures an unbiased 
sampling. Average values calculated with the weighted particles do not depend on the thinning energy, only fluctuations are artificially increased when thinning is applied and depend on the thinning energy. The effect of the Hillas thinning mechanism on the lateral distribution of electrons and positrons from a $10^{19} \mathrm{eV}$ vertical proton shower is shown in Fig. 11. The ratio of particle densities with thinning levels $\varepsilon_{\mathrm{th}}=E_{\mathrm{th}} / E_{\text {prim }}=10^{-6}$ and $10^{-7}$ to a reference density (representing densities without thinning) are plotted. The artificial fluctuations due to thinning, showing up as fluctuations of the curve around 1, are clearly reduced with the smaller thinning level. The simulation done at $\varepsilon_{\mathrm{th}}=10^{-7}$ produced 10 times more output at ground level and required $\approx 8.4$ times more $\mathrm{CPU}$ time than that for $\varepsilon_{\text {th }}=10^{-6}$. The fluctuations are reduced by about a factor 3-4 for the electromagnetic component. For $10^{19} \mathrm{eV}$ showers with $10^{-7}$ thinning computing times still range up to about $10 \mathrm{~h}$ per shower (on a $500 \mathrm{MHz}$ machine) and secondary particles at ground level carry weights of up to $10^{7}$. In its original form, the thinning algorithm depends only on particle energies and the method is very effective close to the shower core, where the particle densities are high. With an array of widely-separated surface detectors, however, most of the detectors sample the air shower in a core distance range in which the particle densities are rather small. Here thinning introduces large artificial fluctuations. To reduce thinning fluctuations the particle selection rule is modified to consider particle energies as well as weights and the core distance.

A practical method for reducing the thinning fluctuations was investigated by Kobal [56]. By limiting the weight to a maximum value $w_{\text {lim }}$ particles with higher weights can be avoided. Particles with weights $\approx w_{\text {lim }}$ are no longer thinned and all their secondaries are tracked. The introduction of
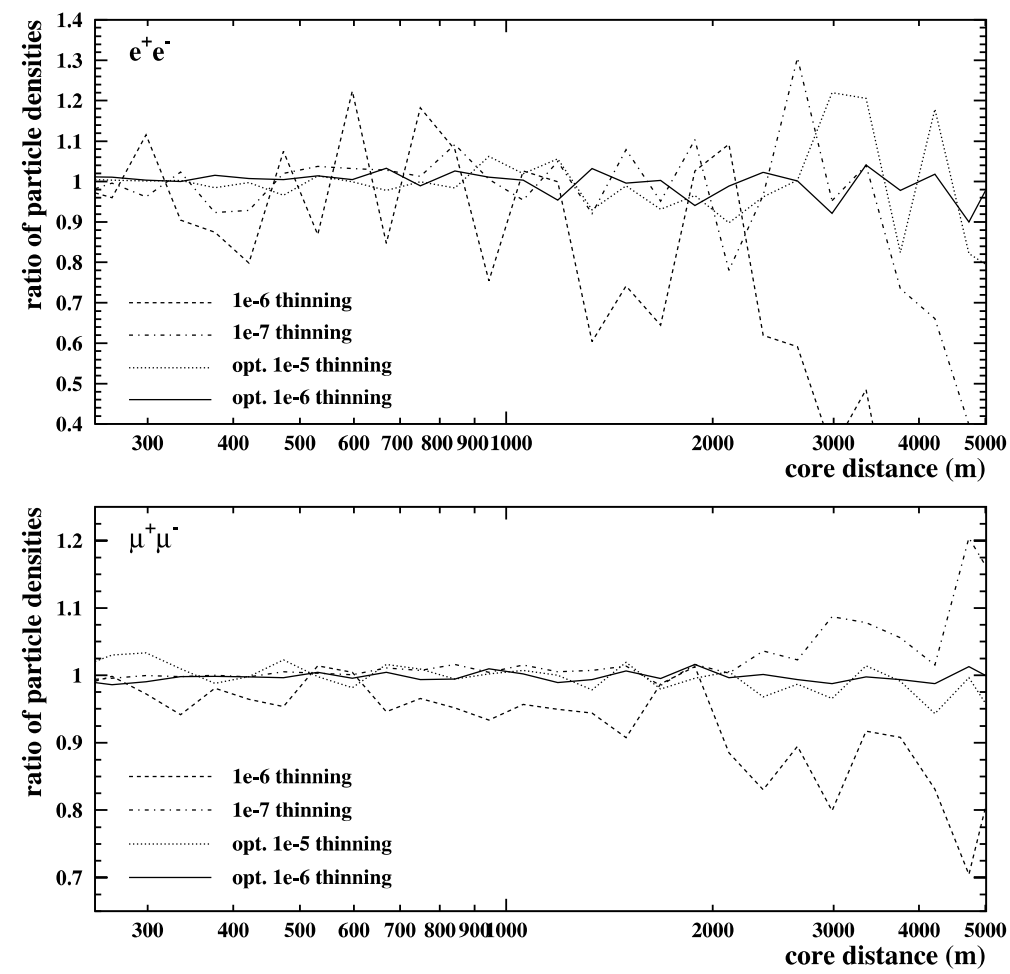

Fig. 11. Effect of the thinning energy on fluctuations of the lateral distribution of $\mathrm{e}^{+} \mathrm{e}^{-}$and $\mu^{+} \mu^{-}$. Eight vertical proton showers of $10^{19}$ $\mathrm{eV}$ have been averaged for each thinning level and divided by a reference lateral distribution. Results for $\varepsilon_{\mathrm{th}}=10^{-6}$ and $10^{-7}$ without weight limitation, and for $\varepsilon_{\mathrm{th}}=10^{-5}$ and $10^{-6}$ with optimum weight limitation are shown. 
a weight limit, while maintaining the thinning energy, increases the computing time and the particle output again. However, thinning energy and weight limit can be optimised such that the sampling fluctuations become minimal for a given computing time. The optimum choice for most cases is $w_{\text {lim }}=E_{\text {prim }}($ in $\mathrm{GeV}) \varepsilon_{\mathrm{th}}$ [56].

For a $10^{19} \mathrm{eV}$ proton shower, for example, the artificial fluctuations for $\varepsilon_{\mathrm{th}}=10^{-7}$ without weight limitation are about $25 \%$ larger than for $\varepsilon_{\mathrm{th}}=10^{-5}$ with optimum weight limitation, and the computing time for the latter is smaller by a factor 4 . To reduce the artificial fluctuations further the thinning level can be reduced. $\varepsilon_{\mathrm{th}}=10^{-6}$ with optimum weight limitation needs twice as long as thinning with $\varepsilon_{\mathrm{th}}=10^{-7}$ without weight limitation, but the artificial fluctuations are reduced by $75 \%$. Thus, to optimise thinning is of great advantage. The artificial fluctuations are about inversely proportional to the square root of the number of particles recorded at ground level.

The computing time is dominated by tracking electromagnetic particles with average energies about 100 times lower than those of muons and hadrons. Consequently, electrons and photons carry weights that are about 100 times higher than the muon and hadron weights. Therefore, thinning and weight limitation can be applied differently to electromagnetic and muonic or hadronic particles, e.g. limiting muons and hadrons to a 100 times lower maximum weight and/or following them to a smaller thinning energy.

An additional trick, the so-called radial thinning, reduces the number of shower particles written to the output file, while still performing the complete simulation. In the very dense region near the centre of the shower, i.e. for $r<r_{0}$, the particle numbers are so large that detectors would be saturated for high-energy showers. The probability to retain a particle is typically chosen to be $\propto\left(r / r_{0}\right)^{k}$ with $r_{0} \approx 150 \mathrm{~m}$ and $k=2 \ldots 6$. This method allows a reduction of information stored by a factor between 2 and 5 , without significant increase of statistical fluctuations for the inner region, and with no loss at all at large core distances, where usually most of the detectors are.

At present thinning seems unavoidable for coping with the huge number of particles in a high- energy air shower. However, a severe disadvantage of thinning becomes apparent when the shower particle lists are used to calculate realistic detector responses, which is necessary to compare simulations with experimental data. It is non-trivial to simulate detector signals and their spread in time from particles that carry large statistical weights, and different methods are being developed to overcome this problem [21].

\section{Characteristics of two simulation programs}

At present two program packages are available to simulate highest-energy air showers. These are CORSIKA (COsmic Ray SImulation for KAscade) $[44,54]$ and AIRES (AIR shower extended simulation) [66]. CORSIKA and AIRES both provide fully four-dimensional MC simulations of proton, photon, and nucleus-induced air shower development in the atmosphere. Both simulate hadronic and electromagnetic interactions, propagate particles through the curved atmosphere, account for the Earth's magnetic field, for decays, energy loss and deflection (and many less important processes), and produce a list of all particles reaching ground level. Both programs assume the same parametrisation of the US Standard Atmosphere as the atmospheric model.

AIRES is originally based on MOCCA [47], but was rewritten and significantly improved and extended. The additions comprise links to the external high-energy hadronic interaction models SIBYLL 1.6 and QGSJET 98, the production of $\mathrm{e}^{+} \mathrm{e}^{-}$pairs and bremsstrahlung by muons, photonuclear reactions, the LPM effect for high-energy $\gamma$ and $\mathrm{e}^{ \pm}$, and the simulation of exotic primaries (e.g. v) [19]. For low-energy hadronic interactions AIRES uses the HSA.

CORSIKA has been developed, over the last 12 years, to become a standard analysis tool for the air shower community. CORSIKA attempts to model the individual processes of the shower development in as great detail as possible, to some extent irrespective of the computing effort needed. It employs proven solutions wherever available. A variety of hadronic models have been linked to CORSIKA and are used and updated to the 
specifications of their respective authors. The hadronic models HDPM [25,44], SIBYLL 2.1 [35], DPMJET 2.5 [64], VENUS [70], QGSJET 01 $[45,50,51]$, and NEXUs $2[33,48]$ are available above $E_{\text {lab }}=80 \mathrm{GeV}$ per nucleon, and GHEISHA [36] and UrQMD [18] for energies below. CORSIKA uses an adapted version of EGS4 [61] for the detailed simulation of electromagnetic interactions, that includes the LPM effect, the production of muon pairs and hadrons by photons, muon bremsstrahlung and $\mathrm{e}^{+} \mathrm{e}^{-}$pair production by $\mathrm{mu}-$ ons. EGS was modified to accommodate the variable atmospheric density, and to compute particle production with double precision. The particles are followed to energies of typically 100 $\mathrm{keV}$. In addition the total energy deposited along the shower axis is recorded. All two- and threebody decays, with branching ratios down to $1 \%$, are modeled kinematically correct and particle tracking and multiple scattering are done in great detail. To account for seasonal and geographical variations CORSIKA permits the choice of a variety of atmospheric density profiles and the definition of new ones.

AIRES uses its own procedures to simulate photo-electric effect, Compton effect, electron bremsstrahlung, $\mathrm{e}^{+} \mathrm{e}^{-}$pair production, and the emission of knock-on electrons similar to what is done in EGS4 or GEANT. Charged particle (multiple) scattering is treated by an effective emulation of Molière's theory with finite nuclear size corrections. The AIRES electromagnetic procedures work down to kinetic energies of $85 \mathrm{keV}$. Particles below this threshold are discarded.

Both programs use a statistical thinning algorithm to keep computing times and particle output at a manageable level. CORSIKA employs optimum thinning following Hillas [46] and Kobal [56] while AIRES uses its own approach [66] that also limits the statistical weights.

AIRES, with the HSA and its own electromagnetic interaction routines, is tuned to be fast. AIRES is about of factor 3.5 faster than CORSIKA when running with comparable (but non-optimum) thinning. For simulations of highest-energy showers with minimum thinning, computing time may be the limiting factor and this difference in speed may prove important. The particle output of AIRES is smaller than that of CORSIKA. Both programs store eight words of output information per particle (i.e. particle id, $\mathrm{p}_{x}$, $\mathrm{p}_{y}, \mathrm{p}_{z}, x, y, t$, weight). CORSIKA stores each word with 32 bits (4 bytes), while AIRES provides the output in its own reduced precision format with about 18 bits/word. This may be of advantage in case a large shower library is produced and the available disk space is limited.

\section{Some results}

Recently the performance of CORSIKA and AIRES has been compared for quantities that are measured in the AUGER experiment [67]. These are the longitudinal shower development, the lateral distribution of particles far from the shower core, energy and time distributions, all for different particle types. Both programs used the QGSJET 98 model for high-energy hadronic interactions $\left(E_{\text {lab }}>80 \mathrm{GeV}\right)$. Averaged results for 100 vertical proton showers of $2 \times 10^{19} \mathrm{eV}$ with thinning at $\varepsilon_{\text {th }}=10^{-7}$ without weight limitation are shown in Figs. 12-15.

Longitudinal shower development. The fluorescence light yield is determined by the energy deposit in the atmosphere, which, in turn, is dominated by the ionization due the numerous charged particles close to the shower axis. Thus, the fluorescence light is closely related to the total number of electrons (and positrons) as a function of depth. This curve, however, is dominated by the high-energy model and how it transfers the initial hadronic energy into the electromagnetic channel. The longitudinal shower development is crucially dependent on the inelastic cross-section and the inelasticity of interactions. Thus, low-energy hadronic and electromagnetic models impose only second-order effects on it.

There is a large difference apparent in $N_{\gamma}$ as function of depth. This is due to the fact, that in CORSIKA upward going particles are discarded. These are predominantly very low-energy (sub $\mathrm{MeV}$ ) photons which contribute less than $2 \%$ to the energy deposit in the atmosphere. The disagreement in $N_{\gamma}$ vanishes almost completely if AIRES discards the upward going particles 

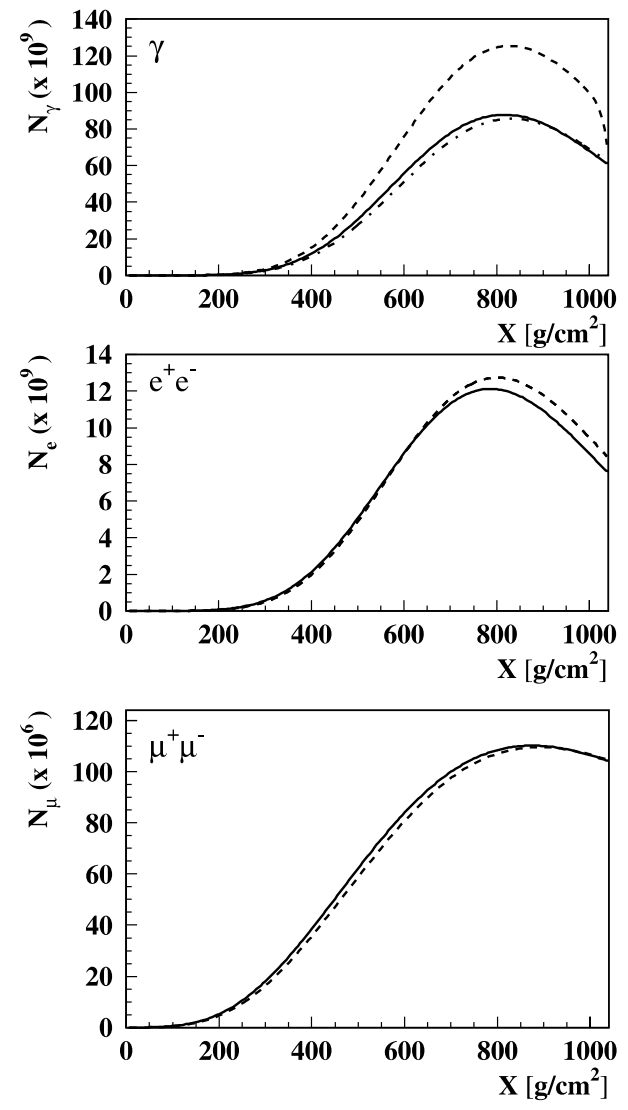

Fig. 12. Longitudinal development of $N_{\gamma}, N_{\mathrm{e}^{ \pm}}\left(E_{\gamma, \mathrm{e}^{+}, \mathrm{e}^{-}} \geqslant 0.1\right.$ $\mathrm{MeV})$ and $N_{\mu^{ \pm}}\left(E_{\mu^{+}, \mu^{-}} \geqslant 100 \mathrm{MeV}\right)$ with atmospheric depth for vertical proton showers of $2 \times 10^{19} \mathrm{eV}$. CORSIKA: solid line, AIRES including (excluding) upward going particles: dashed (dot-dashed) line. For clarity the dot-dashed curves for $\mathrm{e}^{+} \mathrm{e}^{-}$and $\mu^{+}, \mu^{-}$are not shown. They agree with the solid line within $2 \%$.

(dot-dashed line). At sea level the photon number agrees to about $10 \%$.

The predicted evolution of $N_{\mathrm{e}^{+} \mathrm{e}^{-}}$as a function of atmospheric depth agrees well for the two programs, the electron numbers at the maximum of the shower development differ by about $6 \%$, while the mean positions of the maximum differ by about $25 \mathrm{~g} / \mathrm{cm}^{2}$ (see Fig. 12). This difference is also due to the different treatment of upward going particles. If in both programs the upward going particles are discarded the electron longitudinal distributions agree within $2 \%$.

The muon numbers as function of depth, which sensitively depend on details of the hadronic
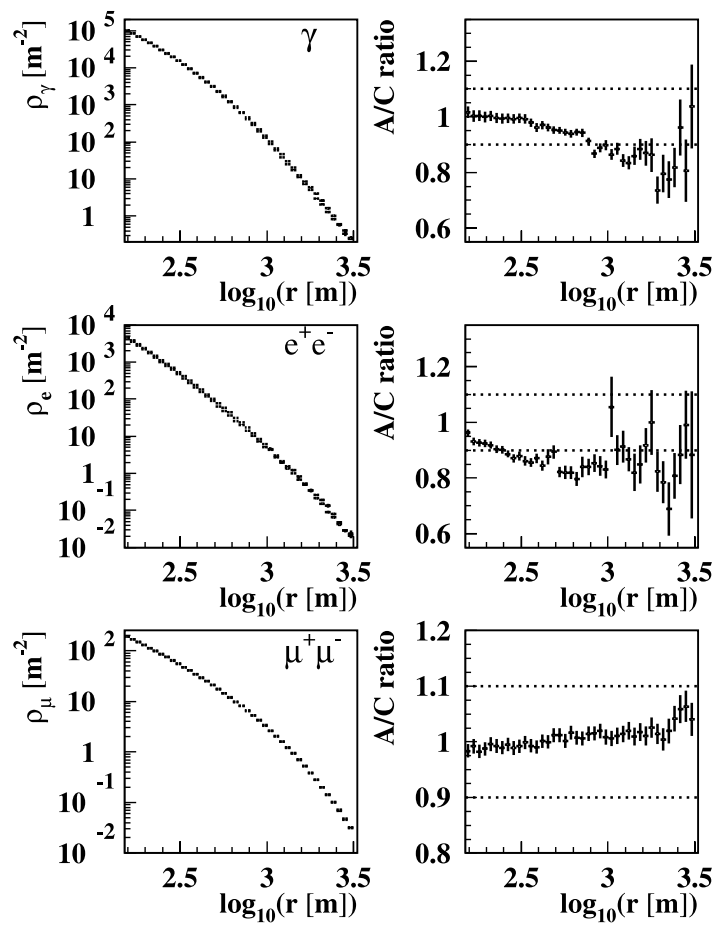

Fig. 13. Lateral particle densities for photons, electrons and muons in vertical $2 \times 10^{19} \mathrm{eV}$ p showers. Left: particle densities. The AIRES and CORSIKA points are virtually on top of each other. Right: density ratios $\rho$ (AIRES) $/ \rho$ (CORSIKA) as function of core distance.

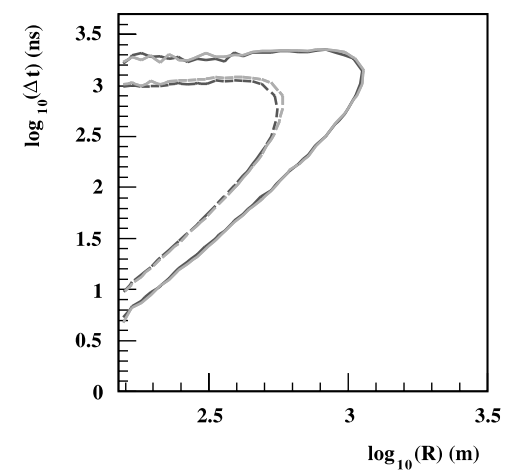

Fig. 14. Particle density contours for photons in the core distance and arrival time plane, for vertical $2 \times 10^{19} \mathrm{eV}$ p showers. CORSIKA: black, AIRES: grey.

models, agree even better. The differences at the shower maximum are about 3\%. 

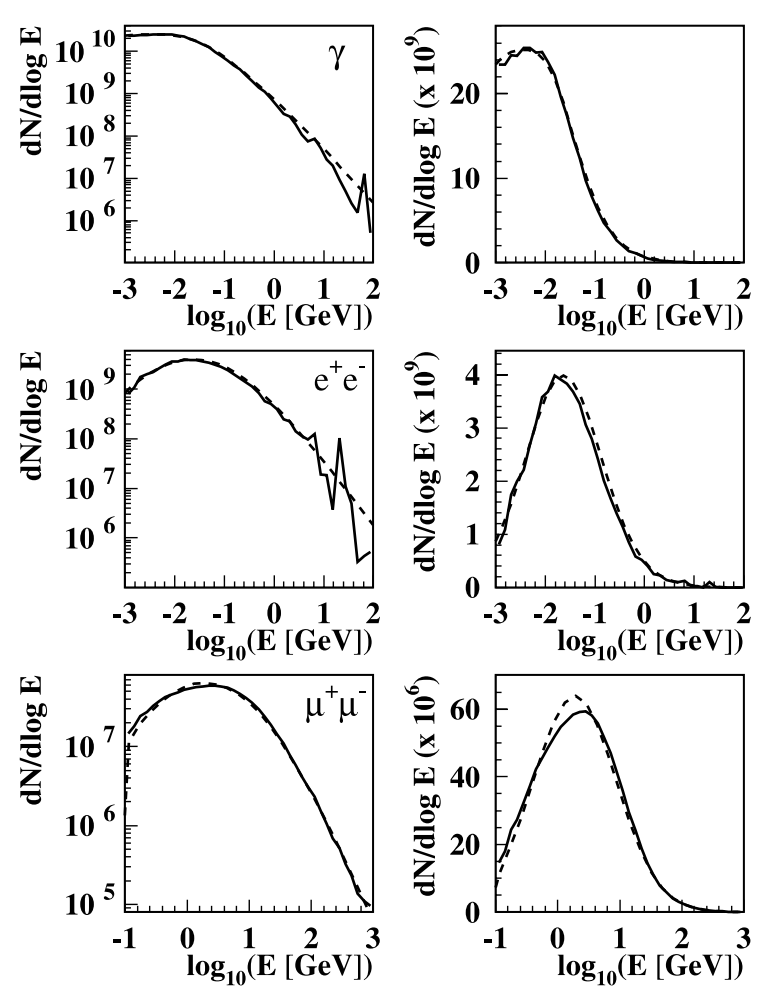

Fig. 15. Energy distributions for photons, electrons and muons. Left: logarithmic abscissa, right: linear abscissa. CORSIKA: (-), AIRES: (-- -).

Lateral distributions. The Auger array detectors measure the density of Cherenkov photons produced by shower particles in water. The array detectors are positioned on a hexagonal grid with $1.5 \mathrm{~km}$ separation. This means that array detectors will rarely be close to the shower core. Typically, particle densities will be recorded in the range $r>300 \mathrm{~m}$. Fig. 13 shows the lateral densities of secondary photons, electrons and muons. The density ratios, $\rho$ (AIRES) $/ \rho$ (CORSIKA), on the right show the differences clearly. With core distance CORSIKA tends to predict higher photon and electron densities, reaching $\approx 20 \%$ for electrons at $\mathrm{km}$ distances. The muon densities agree better. At $r \approx 3 \mathrm{~km}$ a deviation of about $3 \%$ is observed. Fig. 14 shows the photon distribution as function of core distance and arrival time. As expected, the larger the core distance the later the particles arrive on average. The agreement between AIRES and CORSIKA is very good. The agreement between the programs, despite the differences on the microscopic level, demonstrates that particle densities at large core distances are mainly determined by the transverse momenta at particle production and by multiple scattering, and less by details of the low-energy models.

Energy distributions. The density of Cherenkov photons produced in a water tank by shower particles (called the Cherenkov density) is approximately proportional to the energy deposited and, therefore, depends not only on the particle density but also on the energy the particles carry. Electrons and photons are basically absorbed in the water, i.e. deposit all their energy (typically $1-$ $100 \mathrm{MeV}$ ), while muons usually penetrate the tank and release an energy of $\approx 2 \mathrm{MeV} / \mathrm{cm} \times$ their tracklength (typically $240 \mathrm{MeV}$ ). Together with the fact that the muon density decreases more slowly with $r$ than the electron and photon densities, this means that the muon component is dominant at large core distances, as measured by the tank response. Also the energy distribution has a more direct relation to the low-energy hadronic model than have longitudinal or lateral distributions, since the form of the shower is basically determined from the higher-energy interactions. Fig. 15 shows the energy distributions for photons, electrons and muons in a logarithmic and a linear display. The general agreement between AIRES and CORSIKA distributions is good. The most obvious discrepancies (in AIRES with respect to CORSIKA) are a slight excess of photons and electrons with $E>10 \mathrm{GeV}$, and a deformation of the muon spectrum below $3 \mathrm{GeV}$, leading to a deficit for muons with $E<0.5 \mathrm{GeV}$ and an excess for $0.5<E<3 \mathrm{GeV}$. It is rather likely that both discrepancies stem from the low-energy hadronic model, e.g. from the higher $\pi$ yield in the HSA as compared to GHEISHA.

The general agreement between AIRES and CORSIKA in longitudinal, lateral and energy distributions is good. No discrepancies are found beyond the $20 \%$ level.

Models compared to experimental data. Though the comparison of models between each other is an important exercise, an important test is the comparison of simulations with experimental data. This requires a detailed understanding of the 
detector response to the shower particles. A variety of experiments have used modern shower and detector simulation tools and have reported good overall agreement of model simulations with measurements. In the following just three examples from different energy regions will be shown.

The KASCADE experiment [52] records air showers in the energy range $10^{14}$ to $10^{16} \mathrm{eV}$ and measures simultaneously the electromagnetic, muonic and hadronic shower component with good accuracy. This offers a good basis for a variety of model tests $[10,11]$. One of the quantities examined is the ratio of muon to electron number. Fig. 16 shows the measured distribution of the muon to electron ratio $\log _{10} N_{\mu}^{\mathrm{tr}} / \log _{10} N_{\mathrm{e}}$ in showers with $E_{0} \approx 2 \times 10^{15} \mathrm{eV}$. This distribution is compared with CORSIKA/QGSJET 98 simulations for $\mathrm{p}$, $\mathrm{He}, \mathrm{O}$ and $\mathrm{Fe}$ primaries. The relative fractions of

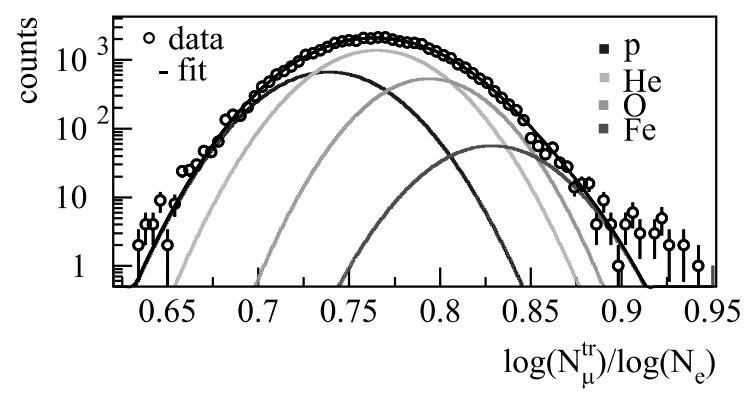

Fig. 16. Distribution of muon to electron ratio $\log _{10} N_{\mu}^{\mathrm{tr}} /$ $\log _{10} N_{\mathrm{e}}$ in showers of energy $\approx 2 \times 10^{15} \mathrm{eV}$. From Ref. [69]. primaries are adjusted to fit the experimental curve. It is a great confirmation of the model calculations that the muon-to-electron ratio of simulated protons fits the left part, and the iron simulations fit, to some extent, the right part of the experimental distribution, without any arbitrary adjustment.

The Haverah Park experiment has measured air showers at energies between $3 \times 10^{17}$ and $10^{20} \mathrm{eV}$. Composition studies in the 1980 s proved to be impossible since models at that time where not able to reproduce the measured lateral distribution of shower particles at all. Recently a sub-set of these data with $3 \times 10^{17} \mathrm{eV}<E<3 \times 10^{18} \mathrm{eV}$ has been re-analysed and interpreted by comparison to CORSIKA/QGSJET 01 simulations [14,15]. Now simulations reproduce the data very well. Fig. 17 shows the signal rise times in water-Cherenkov detectors as a function of core distance. For the core distances shown the simulations for proton and iron do not differ enough to allow a composition estimate, but the fact that the measured data fall in between the simulations for protons and iron indicates that the particle arrival times are well described by the shower models. Fig. 18 shows the distribution of the shape parameter $\eta$, which is used to parametrise the slope of the waterCherenkov signal as a function of core distance and is correlated to the height of the shower maximum. The upper two panels show that the data cannot be described by a pure proton or iron composition. For two different energies the lower
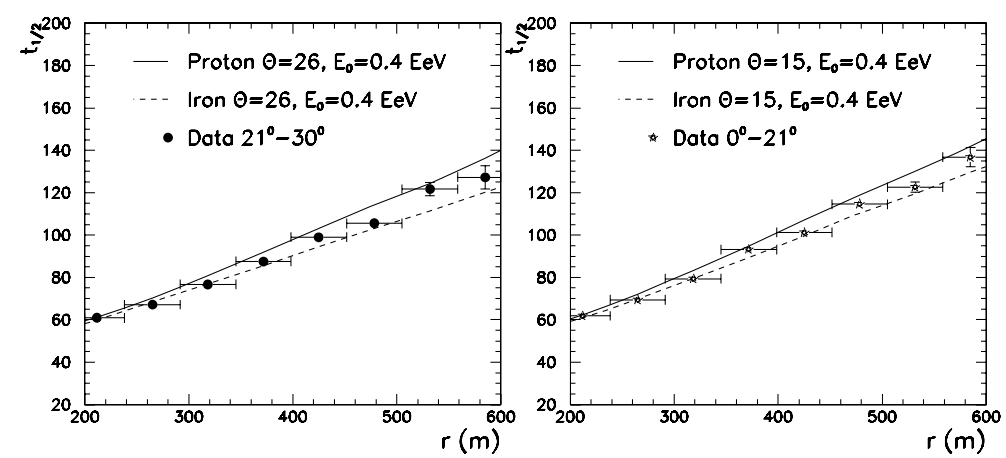

Fig. 17. Signal rise time as function of core distance for Haverah Park data and CORSIKA/QGSJET 01 simulations for two different zenith angles. From Ref. [15]. 

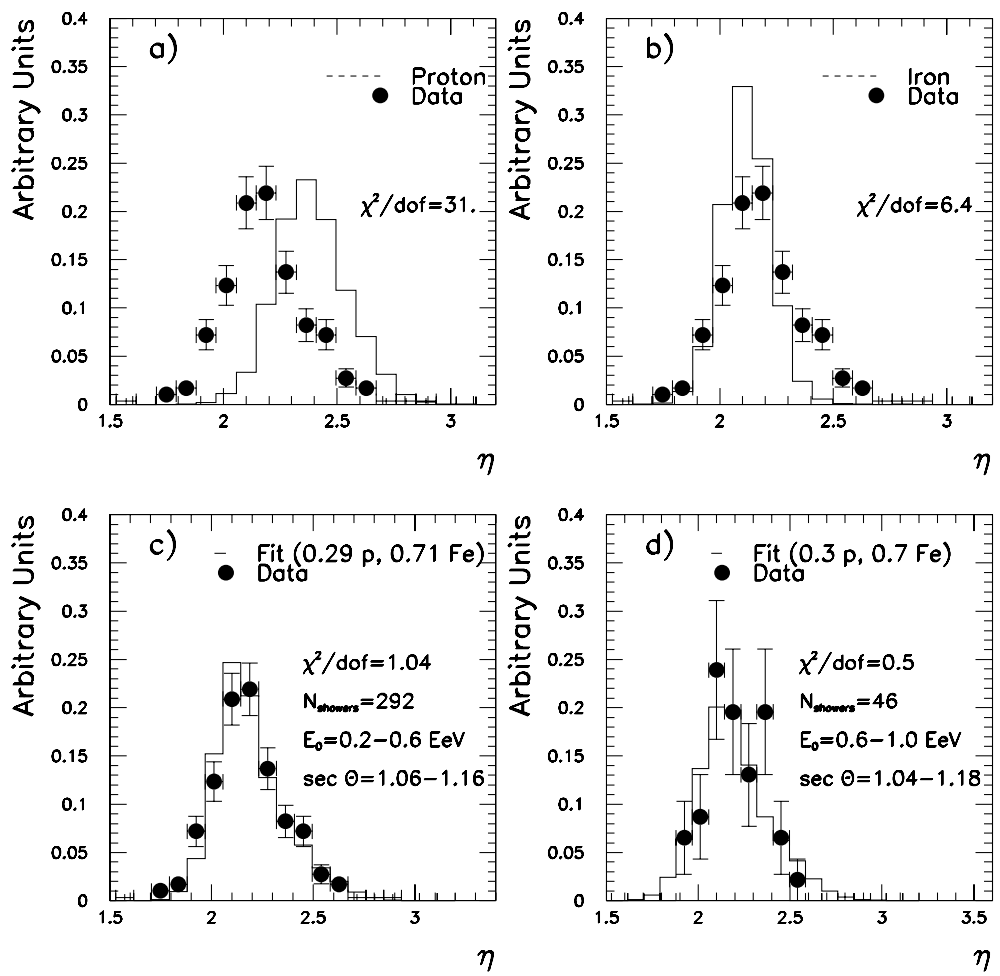

Fig. 18. Distribution of the shape parameter $\eta$ for Haverah Park data and CORSIKA/QGSJET 01 simulations. The solid points represent experimental data, the histograms the simulations. (a), (b) and (c) show results for $E_{0}=0.2-0.6 \mathrm{EeV}$, and (d) for $E_{0}=0.6-1$ EeV. From Ref. [15].

panels demonstrate that a mix of proton and iron yields a good fit to the data.

Finally, even at energies up to $10^{20} \mathrm{eV}$ the air shower models seem to be close to the experimental data. A comparison of AGASA data with CORSIKA/QGSJET 98 simulations [60] shows that the form of the average lateral distributions of muons and of all charged particles agrees well for core distances from 300 to $2000 \mathrm{~m}$.

\section{Summary}

In the last decade much has changed in air shower simulations. Through better understanding of the relevant hadronic interactions and the massive increase in computer performance very elaborate MC models became feasible that track individual particles through atmosphere and de- tectors and simulate all their interactions with matter in great detail. Predictions of air shower programs have become much more quantitative and describe the experimental data sufficiently well for data analysis and the design of new experiments.

The weakest point in the simulations are the high-energy soft hadronic interactions, which are not well examined at accelerators so far, and the extrapolation to energies much beyond those available at accelerators. The most successful models are based on the Gribov-Regge theory of multi-Pomeron exchange. There is a clear trend of convergence between different hadronic interaction models, due to objective improvement of the physics input. However, a level of systematic uncertainty of about $20 \%$ is likely to persist, since many of the input data, on which the models are built, appear to have uncertainities of this size. 
Once observed, larger inconsistencies between data and simulations offer a possibility to improve the model assumptions. The calorimetric measurement of the shower energy via the fluorescence detectors in coincidence with the charged particle measurements in the Auger experiment will assist with stringent tests of the shower models and promises the determination of an absolute energy scale as well as setting constraints on the shower models.

Within the Auger collaboration the two programs CORSIKA and AIRES are used. They account for all processes necessary for air showers simulations up to $10^{21} \mathrm{eV}$. The programs complement each other in the sense that CORSIKA uses more elaborate interaction models and AIRES is faster. CORSIKA and AIRES agree to better than $20 \%$ for the basic shower parameters observed by the Pierre Auger Observatory.

\section{Acknowledgements}

We are grateful for comments on an earlier version of this manuscript to R. Engel, S. Ostapchenko, and A.A. Watson. JK, DH and MR acknowledge support for a British-German Academic Research Collaboration from The British Council and the DAAD.

\section{References}

[1] CDF Collaboration, F. Abe et al., Phys. Rev. D 50 (1994) 5550.

[2] T. Abu-Zayyad et al., Phys. Rev. Lett. 84 (2000) 4276.

[3] T. Abu-Zayyad et al., Astrophys. J. 557 (2001) 686.

[4] H1 Collaboration, C. Adloff et al., Nucl. Phys. B 497 (1997) 3 .

[5] EAS-TOP Collaboration, M. Aglietta et al., Proc. 26th Int. Cosmic Ray Conf., Salt Lake City, USA, HE 1.3.04, 1999.

[6] H1 Collaboration, T. Ahmed et al., Phys. Lett. B 299 (1993) 374

[7] UA5 Collaboration, G.J. Alner et al., Z. Phys. C 33 (1986) 1.

[8] E710 Collaboration, N.A. Amos et al., Phys. Rev. Lett. 68 (1992) 2433.

[9] L.A. Anchordoqui et al., Phys. Rev. D 59 (1999) 094003.

[10] KASCADE Collaboration, T. Antoni et al., J. Phys. G: Nucl. Part. Phys. 25 (1999) 2161.
[11] KASCADE Collaboration, T. Antoni et al., J. Phys. G: Nucl. Part. Phys. 27 (2001) 1785.

[12] HEGRA Collaboration, F. Arqueros et al., Astron. Astrophys. 359 (2000) 682.

[13] Auger Collaboration, Auger Project Design Report, FNAL, 1997.

[14] M. Ave et al., astro-ph/0112253, Astropart. Phys., in press.

[15] M. Ave et al., astro-ph/0203150, Astropart. Phys., in press.

[16] E811 Collaboration, C. Avila et al., Phys. Lett. B 445 (1999) 419.

[17] R.M. Baltrusaitis et al., Phys. Rev. Lett. 52 (1984) 1380.

[18] S.A. Bass et al., Prog. Part. Nucl. Phys. 41 (1998) 225; M. Bleicher et al., J. Phys. G: Nucl. Part. Phys. 25 (1999) 1859.

[19] X. Bertou et al., Astropart. Phys. 17 (2002) 183.

[20] X. Bertou, P. Billoir, Pierr Auger technical note, GAP 1998-049, 1998.

[21] P. Billoir, Reconstruction of Showers with the Ground Array, Pierre Auger technical note, GAP 2000-025, 2000.

[22] D.J. Bird et al., Phys. Rev. Lett. 71 (1993) 3401.

[23] M.M. Block, F. Halzen, T. Stanev, Phys. Rev. D 62 (2000) 077501.

[24] R. Brun et al., GEANT 3, Detector Description and Simulation Tool, CERN, Program Library CERN, 1993.

[25] J.N. Capdevielle, J. Phys. G: Nucl. Part. Phys. 15 (1989) 909.

[26] K.J. Capelle et al., Astropart. Phys. 8 (1998) 321.

[27] C. Caso et al., Review of particle physics (Particle Data Group), Eur. Phys. J. C 3 (1998) 1.

[28] A.N. Cillis, H. Franchiotti, C.A. Garcia Canal, S.J. Sciutto, Phys. Rev. D 59 (1999) 113012.

[29] A.N. Cillis, S.J. Sciutto, Phys. Rev. D 64 (2001) 013010.

[30] B. Dawson et al., Astropart. Phys. 9 (1998) 331.

[31] ZEUS Collaboration, M. Derrick et al., Phys. Lett. B 293 (1992) 465.

[32] J.E. Dickinson et al., Proc. 26th Int. Cosmic Ray Conf., Salt Lake City, USA, vol. 3, 1999, pp. 136.

[33] H.J. Drescher et al., Phys. Rep. 350 (2001) 93.

[34] M.N. Dyakonov et al., Proc. 23rd Int. Cosmic Ray Conf., Calgary, Canada, vol. 4, 1993, pp. 303.

[35] R. Engel, T.K. Gaisser, T. Stanev, Proc. 26th Int. Cosmic Ray Conf., Salt Lake City, USA, vol. 1, 1999, pp. 415; R. Engel et al., in preparation.

[36] H. Fesefeldt, The Simulation of Hadronic Showers-Physics and Application, Rheinisch-Westfälische Technische Hochschule, Aachen PITHA 85/02, 1985.

[37] R.S. Fletcher, T.K. Gaisser, P. Lipari, T. Stanev, Phys. Rev. D 50 (1994) 5710;

J. Engel, T.K. Gaisser, P. Lipari, T. Stanev, Phys. Rev. D 46 (1992) 5013.

[38] J.W. Fowler et al., Astropart. Phys. 15 (2001) 49.

[39] G.M. Frichter, T.K. Gaisser, T. Stanev, Phys. Rev. D 50 (1997) 3135

[40] T.K. Gaisser et al., Phys. Rev. D 47 (1993) 1919.

[41] R.J. Glauber, G. Matthiae, Nucl. Phys. B 21 (1970) 135.

[42] L.V. Gribov et al., Phys. Rep. 100 (1983) 1.

[43] R. Harr et al., Phys. Lett. B 401 (1997) 176. 
[44] D. Heck et al., CORSIKA: A Monte Carlo Code to Simulate Extensive Air Showers, Forschungszentrum Karlsruhe FZKA 6019, 1998.

[45] KASCADE Collaboration, D. Heck et al., Proc. 27th Int. Cosmic Ray Conf., Hamburg, Germany, HE 1.3 233, 2001.

[46] A.M. Hillas, Proc. 17th Int. Cosmic Ray Conf., Paris, France, vol. 8, 1981, pp. 193;

A.M. Hillas, Proc. 19th Int. Cosmic Ray Conf., La Jolla, USA, vol. 1, 1985, pp. 155.

[47] A.M. Hillas, Nucl. Phys. B (Proc. Suppl.) 52B (1997) 29.

[48] M. Hladik et al., Phys. Rev. Lett. 86 (2001) 3506.

[49] M. Honda et al., Phys. Rev. Lett. 70 (1993) 525.

[50] N.N. Kalmykov, S.S. Ostapchenko, Yad. Fiz. 56 (1993) 105 ;

N.N. Kalmykov, S.S. Ostapchenko, Phys. Atom. Nucl. 56 (3) (1993) 346;

N.N. Kalmykov, S.S. Ostapchenko, A.I. Pavlov, Bull. Russ. Acad. Sci. (Physics) 58 (1994) 1966.

[51] N.N. Kalmykov, S.S. Ostapchenko, A.I. Pavlov, Nucl. Phys. B (Proc. Suppl.) 52B (1997) 17.

[52] KASCADE Collaboration, H.O. Klages et al., Nucl. Phys. B (Proc. Suppl.) 52B (1997) 92.

[53] J. Knapp, Nucl. Phys. B (Proc. Suppl.) 75A (1999) 89.

[54] J. Knapp, D. Heck, Extensive Air Shower Simulation with CORSIKA: A User's Manual, Kernforschungszentrum Karlsruhe KfK 5196 B, 1993; for an up-to-date version see http://www-ik3.fzk.de/heck/corsika.

[55] S. Knurenko et al., Proc. 27th Int. Cosmic Ray Conf., Hamburg, Germany, HE 1.3, 2001, pp. 177.
[56] Pierre Auger Collaboration, M. Kobal, Astropart. Phys. 15 (2001) 259.

[57] L.D. Landau, I.Ya. Pomeranchuk, Dokl. Akad. Nauk SSSR 92 (1953) 535-735;

A.B. Migdal, Phys. Rev. 103 (1956) 1811;

E. Konishi et al., J. Phys. G: Part. Phys. 17 (1991) 719.

[58] B. McBreen, C.J. Lambert, Phys. Rev. D 24 (1981) 2536.

[59] H.H. Mielke et al., J. Phys. G: Nucl. Part. Phys. 20 (1994) 637.

[60] M. Nagano et al., Astropart. Phys. 13 (2000) 277.

[61] W.R. Nelson, H. Hirayama, D.W.O. Rogers, The EGS4 code system, Stanford Linear Accelerator Center SLAC 265 (1985).

[62] C.L. Pryke, Astropart. Phys. 14 (2001) 319.

[63] J. Ranft, Phys. Rev. D 51 (1995) 64.

[64] J. Ranft, preprints hep-ph/9911213 and hep-ph/9911232, 1999.

[65] M. Risse et al., Proc. 27th Int. Cosmic Ray Conf., Hamburg, Germany, HE 1.5, 2001, pp. 522.

[66] S.J. Sciutto, preprint astro-ph/9911331, 1999; see also http://www.fisica.unlp.edu.ar/auger/aires.

[67] S.J. Sciutto, J. Knapp, D. Heck, Proc. 27th Int. Cosmic Ray Conf., Hamburg, Germany, HE 1.5, 2001, pp. 526.

[68] S.P. Swordy, D.B. Kieda, Astropart. Phys. 13 (2000) 137.

[69] KASCADE Collaboration, J. Weber et al., Proc. 26th Int. Cosmic Ray Conf., Salt Lake City, USA, vol. 1, 1999, pp. 341 .

[70] K. Werner, Phys. Rep. 232 (1993) 87.

[71] G.B. Yodh et al., Phys. Rev. D 27 (1983) 1183. 